\title{
STOCHASTIC LINEAR QUADRATIC OPTIMAL CONTROL PROBLEMS FOR MEAN-FIELD STOCHASTIC EVOLUTION EQUATIONS*
}

\author{
QI L $\ddot{U}^{* *}$
}

\begin{abstract}
We study a linear quadratic optimal control problem for mean-field stochastic evolution equation with the assumption that all the coefficients concerned in the problem are deterministic. We show that the existence of optimal feedback operators is equivalent to that of regular solution to the system which is coupled by two Riccati equations and an explicit formula of the optimal feedback control operator is given via the regular solution. We also show that the mentioned Riccati equations admit a unique strongly regular solution when the cost functional is uniformly convex.
\end{abstract}

Mathematics Subject Classification. 93E20, 49N10, 49N35.

Received April 11, 2020. Accepted November 18, 2020.

\section{INTRODUCTION}

\subsection{Some literature}

Mean field approximation is extremely useful when describing macroscopic phenomena from microscopic overviews in many areas of science. Typical examples are the study of turbulence, gauge field, plasma physics, quantum chemistry, tumor growth modeling, etc. (e.g. [21]). Over the last years, due to various applications in economics, finance and physics, there has been a growing interest in control problems for mean-field stochastic differential equations (MF-SDEs for short). The interested readers are referred to $[5,7-9]$ and the rich references therein.

Similar to MF-SDEs, Mean-field stochastic evolution equations (MF-SEEs for short) have important applications. Two of them are in order as follows and more examples can be found in $[2,12]$.

1. The population of species in complex environment can be modeled by stochastic parabolic equations (e.g. [11]). In real world, there are a great many species living in the same area, each being influenced by the others. Then a system of a great number of coupled stochastic parabolic equations is used to describe these populations. In practice, a mean-field stochastic parabolic equations is a good simplification of that stochastic parabolic system just mentioned.

* The research of this author is partially supported by NSF of China under grants 11971334, 11931011 and 12025105 , and the Chang Jiang Scholars Program from the Chinese Education Ministry.

Keywords and phrases: Mean-field stochastic evolution equation, linear quadratic optimal control problem, optimal feedback operator, Riccati equation.

School of Mathematics, Sichuan University, Chengdu 610064, Sichuan Province, PR China.

** Corresponding autjor: lu@scu.edu.cn 
2. Stochastic parabolic equations can be used to describe the propagation of the electric potential in a neuron (e.g. [23]). Usually, there are lots of neurons connected with each other. To describe such phenomena, one should use a great number of coupled stochastic parabolic equations. To simplify this system, one can introduce a mean-field stochastic parabolic equation.

Compared with the abundant literature on MF-SDEs, control problems for MF-SEEs received very little attention. To the best of our knowledge, $[2,12,22]$ are the only papers in dealing with optimal control problems of MF-SEEs, in which the authors formulate sufficient and necessary conditions for optimal control problems of MF-SEEs and find optimal open-loop controls.

In this paper, we consider the linear quadratic control problem (LQ problem for short) for MF-SEEs. Due to the elegant mathematical structure and important applications, LQ problems for different kinds of control systems are investigated extensively in the literature (e.g. $[3,4,13,16,18,29])$. Particularly, we refer the readers to $[1,14,25-28]$ and the rich references therein for some recent interesting works for LQ problems of MF-SDEs. However, as far as we know, [22] is the only one published paper addressing the LQ problem for MF-SEEs, in which the auhtors give a characterization of the optimal open-loop controls.

Compared with the LQ problem for stochastic evolution equations (SEEs for short) (e.g. [16]), some new phenomena appear. For example, one needs to introduce a coupled system of Riccati equations to characterize optimal feedback controls. This coupled system is much more complex than a single Riccati equation arising from LQ problems for SEEs. Then one has to find some new technique to study it.

Compared with LQ problems for MF-SDEs (e.g.[1, 14, 19, 26-28]), some new difficulties arise in dealing with infinite dimensional settings. For example, the operator-valued equations are more complicated than the matrix-valued equations. Then one cannot simply mimic the method of solving LQ problems for MF-SDEs to get the expected results.

\subsection{Notations}

In this subsection, we introduce some notations to be used in the sequel.

Let $(\Omega, \mathcal{F}, \mathbb{F}, \mathbb{P})$ be a complete filtered probability space on which a standard one-dimensional Brownian motion $W=\{W(t)\}_{t \geq 0}$ is defined, where $\mathbb{F}=\left\{\mathcal{F}_{t}\right\}_{t \geq 0}$ is the natural filtration of $W$, augmented by all the $\mathbb{P}$-null sets in $\mathcal{F}$.

Let $T>0$ be a fixed time constant. For any $t \in[0, T)$ and a Banach space $\mathbb{H}$, let

$$
\begin{aligned}
& L_{\mathcal{F}_{t}}^{2}(\Omega ; \mathbb{H})=\{\xi: \Omega \rightarrow\left.\mathbb{H} \mid \xi \text { is } \mathcal{F}_{t} \text {-measurable, } \mathbb{E}|\xi|_{\mathbb{H}}^{2}<\infty\right\} \\
& C_{\mathbb{F}}\left([t, T] ; L^{2}(\Omega ; \mathbb{H})\right)=\{\varphi:[t, T] \times \Omega \rightarrow \mathbb{H} \mid \varphi(\cdot) \text { is } \mathbb{F} \text {-adapted, the map } \\
&\left.\varphi:[t, T] \rightarrow L_{\mathcal{F}_{T}}^{2}(\Omega ; \mathbb{H}) \text { is continuous }\right\}, \\
& L_{\mathbb{F}}^{p}\left(\Omega ; L^{q}(t, T ; \mathbb{H})\right)=\{\varphi:[t, T] \times \Omega \rightarrow \mathbb{H} \mid \varphi(\cdot) \text { is } \mathbb{F} \text {-progressively measurable, } \\
& \\
&\left.\mathbb{E}\left(\int_{t}^{T}|\varphi(s)|_{\mathbb{H}}^{q} d s\right)^{\frac{p}{q}}<\infty\right\}
\end{aligned}
$$

where $1 \leq p, q<\infty$. We simply use the symbol $L_{\mathbb{F}}^{p}(t, T ; \mathbb{H})$ to denote $L_{\mathbb{F}}^{p}\left(\Omega ; L^{p}(t, T ; \mathbb{H})\right)$ if $p=q$. .

Let $\mathbb{H}_{1}$ and $\mathbb{H}_{2}$ be two Banach spaces. Write $\mathcal{L}\left(\mathbb{H}_{1} ; \mathbb{H}_{2}\right)$ for the space of all bounded linear operators from $\mathbb{H}_{1}$ to $\mathbb{H}_{2}$. For simplicity, we denote $\mathcal{L}\left(\mathbb{H}_{1} ; \mathbb{H}_{1}\right)$ by $\mathcal{L}\left(\mathbb{H}_{1}\right)$. If $\mathbb{H}$ is a Hilbert space, we write $\mathbb{S}(\mathbb{H})$ for the set of all self-adjoint bounded linear operators on $\mathbb{H}$. Clearly, $\mathbb{S}(\mathbb{H}) \subset \mathcal{L}(\mathbb{H})$ is a closed subspace. For $M, N \in \mathbb{S}(\mathbb{H})$, the notation $M \geq N$ (resp. $M>N$ ) indicates that $M-N$ is positive semi-definite (resp. positive definite). For any $\mathbb{S}(\mathbb{H})$-valued measurable function $F$ on $[t, T]$, we write $F \geq 0($ resp. $F>0, F \gg 0)$ if $F(s) \geq 0($ resp. $F(s)>0$, $F(s) \geq \delta I$ for some $\delta>0)$ for a.e. $s \in[t, T]$. 
Denote by $C_{\mathcal{S}}([t, T] ; \mathbb{S}(\mathbb{H}))$ the set of all strongly continuous mappings $F:[t, T] \rightarrow \mathbb{S}(\mathbb{H})$, that is, $F(\cdot) \eta$ is continuous on $[t, T]$ for each $\eta \in H$. If $F \in C_{\mathcal{S}}([t, T] ; \mathbb{S}(\mathbb{H}))$, then, by the Uniform Boundedness theorem, the quantity

$$
|F|_{C_{\mathcal{S}}([t, T] ; \mathbb{S}(\mathbb{H}))} \triangleq \sup _{s \in[t, T]}|F(s)|_{\mathcal{L}(\mathbb{H})}
$$

is finite, and $C_{\mathcal{S}}([t, T] ; \mathbb{S}(\mathbb{H}))$ is a Banach space with this norm (see [4], Part IV, Chapter 1 for the detail).

\subsection{Formulation of the control problem}

Let $H$ and $U$ be two separable Hilbert spaces. Let $A: D(A) \rightarrow H$ be the generator of a $C_{0}$-semigroup $\left\{e^{A t}\right\}_{t \geq 0}$ on $H$. Consider the following controlled MF-SEE:

$$
\left\{\begin{aligned}
\mathrm{d} x(s)= & {\left[\left(A+A_{1}(s)\right) x(s)+\widehat{A}(s) \mathbb{E} x(s)+B(s) u(s)+\widehat{B}(s) \mathbb{E} u(s)\right] \mathrm{d} s } \\
& +(C(s) x(s)+\widehat{C}(s) \mathbb{E} x(s)+D(s) u(s)+\widehat{D}(s) \mathbb{E} u(s)) \mathrm{d} W(s) \quad \text { in }(t, T], \\
x(t)=\xi &
\end{aligned}\right.
$$

where $\xi \in L_{\mathcal{F}_{t}}^{2}(\Omega ; H)$ and

$$
\begin{aligned}
& A_{1}(\cdot), \widehat{A}(\cdot) \in L^{1}(0, T ; \mathcal{L}(H)), \quad B(\cdot), \widehat{B}(\cdot) \in L^{2}(0, T ; \mathcal{L}(U ; H)), \\
& C(\cdot), \widehat{C}(\cdot) \in L^{2}(0, T ; \mathcal{L}(H)), \quad D(\cdot), \widehat{D}(\cdot) \in L^{\infty}(0, T ; \mathcal{L}(U ; H)) .
\end{aligned}
$$

In (1.1), $u(\cdot)$ is the control and $x(\cdot)=x(\cdot ; t, \xi, u)$ is the state. Each $u(\cdot) \in \mathcal{U}[t, T] \triangleq L_{\mathbb{F}}^{2}(t, T ; U)$ is called an admissible control (on $[t, T]$ ). In what follows, to simplify notations, the time variable $s$ (or $t$ ) is suppressed in $x, A_{1}, B, C, D$, etc.

A standard argument via the Banach fixed point theorem shows that for any $(t, \xi) \in[0, T) \times L_{\mathcal{F}_{t}}^{2}(\Omega ; H)$ and $u(\cdot) \in \mathcal{U}[t, T]$, the control system (1.1) admits a unique mild solution

$$
x(\cdot) \equiv x(\cdot ; t, \xi, u(\cdot)) \in C_{\mathbb{F}}\left([t, T] ; L^{2}(\Omega ; H)\right) .
$$

Define the cost functional for (1.1) by

$$
\begin{aligned}
\mathcal{J}(t, \xi ; u(\cdot)) \triangleq \mathbb{E}[ & \langle G x(T), x(T)\rangle+\langle\widehat{G} \mathbb{E} x(T), \mathbb{E} x(T)\rangle+\int_{t}^{T}(\langle Q x, x\rangle+\langle R u, u\rangle) \mathrm{d} s \\
& \left.+\int_{t}^{T}(\langle\widehat{Q} \mathbb{E} x, \mathbb{E} x\rangle+\langle\widehat{R} \mathbb{E} u, \mathbb{E} u\rangle) \mathrm{d} s\right],
\end{aligned}
$$

where

$$
Q(\cdot), \widehat{Q}(\cdot) \in L^{1}(0, T ; \mathbb{S}(H)), \quad R(\cdot), \widehat{R}(\cdot) \in L^{\infty}(0, T ; \mathbb{S}(U)), \quad G, \widehat{G} \in \mathbb{S}(H)
$$

Here and in what follows, if there is no danger of confusion, we use $\langle\cdot, \cdot\rangle$ to denote the inner product in different Hilbert spaces.

Consider the following mean-field stochastic linear quadratic optimal control problem:

Problem (MF-SLQ). For any given $(t, \xi) \in[0, T) \times L_{\mathcal{F}_{t}}^{2}(\Omega ; H)$, find a control $\bar{u}(\cdot) \in \mathcal{U}[t, T]$ such that

$$
\mathcal{J}(t, \xi ; \bar{u}(\cdot))=\inf _{u(\cdot) \in \mathcal{U}[t, T]} \mathcal{J}(t, \xi ; u(\cdot)) .
$$


Every $\bar{u}(\cdot) \in \mathcal{U}[t, T]$ satisfying (1.4) is called an optimal control of Problem (MF-SLQ) with the initial state $\xi$, and $\bar{x}(\cdot) \equiv \bar{x}(\cdot ; t, \xi, \bar{u}(\cdot))$ is the corresponding optimal state.

We are to present a necessary and sufficient condition for the optimal control. To this end, we introduce the following mean-field forward-backward stochastic evolution equation (MF-FBSEE, for short):

$$
\begin{cases}\mathrm{d} x(s)=\left[\left(A+A_{1}\right) x+\widehat{A} \mathbb{E} x+B u+\widehat{B} \mathbb{E} u\right] \mathrm{d} s+(C x+\widehat{C} \mathbb{E} x+D u+\widehat{D} \mathbb{E} u) \mathrm{d} W(s) & \text { in }(t, T], \\ d y(s)=-\left[\left(A+A_{1}\right)^{*} y+\widehat{A}^{*} \mathbb{E} y+C^{*} Y+\widehat{C}^{*} \mathbb{E} Y+Q x+\widehat{Q} \mathbb{E} x\right] \mathrm{d} s+Y \mathrm{~d} W(s) & \text { in }[t, T), \\ x(t)=\xi, \quad y(T)=G x(T)+\widehat{G} \mathbb{E} x(T) . & \end{cases}
$$

The well-posedness of the first equation in (1.5) can be obtained by standard fixed point argument. Once $x(\cdot)$ is obtained, we can employ the same tequenique to solve the second one in (1.5).

Theorem 1.1. Let $(t, \xi) \in[0, T) \times L_{\mathcal{F}_{t}}^{2}(\Omega ; H)$ be given. Let $u(\cdot) \in \mathcal{U}[t, T]$ and $(x(\cdot), y(\cdot), z(\cdot))$ be the solution to (1.5). Then $u(\cdot)$ is an optimal control of Problem (MF-SLQ) with the initial state $\xi$ if and only if

$$
\mathcal{J}(t, 0 ; u(\cdot)) \geq 0, \quad \forall u(\cdot) \in \mathcal{U}[t, T]
$$

and

$$
B^{*} y+D^{*} Y+R u+\widehat{B}^{*} \mathbb{E} y+\widehat{D}^{*} \mathbb{E} Y+\widehat{R} \mathbb{E} u=0, \quad \text { a.e. } s \in[t, T], \mathbb{P} \text {-a.s. }
$$

Remark 1.2. In [22], an analogous result to Theorem 1.1 is proved under some other kind of conditions.

By Theorem 1.1, one only needs to solve (1.5)-(1.7) to find the optimal control. However, the control obtained in this way is an open-loop one. For optimal control problems, it is important to find a feedback optimal control. This is particularly important in practical applications since the corresponding control strategy is kept robust with respect to (small) perturbation/disturbance.

We need the following notion.

For $t \in[0, T)$, let

$$
\mathscr{F}[t, T]=L^{2}(t, T ; \mathcal{L}(H ; U)) \times L^{2}(t, T ; \mathcal{L}(H ; U)) .
$$

A pair $(\Theta(\cdot), \widehat{\Theta}(\cdot)) \in \mathscr{F}[t, T]$ is called a feedback operator of Problem (MF-SLQ) on $[t, T]$. For any $(\Theta(\cdot), \widehat{\Theta}(\cdot)) \in$ $\mathscr{F}[t, T]$ and $\xi \in L_{\mathcal{F}_{t}}^{2}(\Omega ; H)$, let $x(\cdot) \equiv x(\cdot ; t, \xi, \Theta(\cdot), \widehat{\Theta}(\cdot))$ be the solution of the following closed-loop system:

$$
\left\{\begin{aligned}
\mathrm{d} x= & \left\{\left(A+A_{1}+B \Theta\right) x+[\widehat{A}+B \widehat{\Theta}+\widehat{B}(\Theta+\widehat{\Theta})] \mathbb{E} x\right\} \mathrm{d} s \\
& +\{(C+D \Theta) x+[\widehat{C}+D \widehat{\Theta}+\widehat{D}(\Theta+\widehat{\Theta})] \mathbb{E} x\} \mathrm{d} W(s) \quad \text { in }(t, T], \\
x(t)= & \xi
\end{aligned}\right.
$$

Definition 1.3. A feedback operator $(\bar{\Theta}(\cdot), \overline{\widehat{\Theta}}(\cdot)) \in \mathscr{F}[t, T]$ is optimal if

$$
\mathcal{J}(t, \xi ; \bar{\Theta}(\cdot) \bar{x}(\cdot)+\overline{\widehat{\Theta}}(\cdot) \mathbb{E}[\bar{x}(\cdot)]) \leq \mathcal{J}(t, \xi ; u(\cdot)), \quad \forall \xi \in L_{\mathcal{F}_{t}}^{2}(\Omega ; H), \forall u \in \mathcal{U}[t, T]
$$

where $\bar{x}(\cdot)$ solves $(1.8)$ with $(\Theta(\cdot), \widehat{\Theta}(\cdot))$ replaced by $(\bar{\Theta}(\cdot), \widehat{\Theta}(\cdot))$. If an optimal feedback operator (uniquely) exists on $[t, T]$, Problem (MF-SLQ) is said to be (uniquely) closed-loop solvable on $[t, T]$.

The main problem studied in this paper is as follows:

Does the optimal feedback operator exist and how to characterize it? 


\subsection{Main results}

Before introducing the Riccati equations, we first recall the definition of the generalized pseudo inverse of an operator. More details can be found in [6].

Definition 1.4. Let $H_{1}$ be a Hilbert space. For $F \in \mathcal{L}\left(H_{1}\right)$, a generalized pseudo inverse of $F$ is defined as a linear operator $F^{\dagger}: \mathcal{D}\left(F^{\dagger}\right) \rightarrow H_{1}$ satisfying the following four criteria:

$$
F F^{\dagger} F=F, \quad F^{\dagger} F F^{\dagger}=F^{\dagger}, \quad\left(F F^{\dagger}\right)^{*}=F F^{\dagger}, \quad\left(F^{\dagger} F\right)^{*}=F^{\dagger} F .
$$

When $F$ is injective, $F^{\dagger}$ is a left inverse of $F$. When $F$ is surjective, $F^{\dagger}$ is a right inverse of $F$. When $F$ is self-adjoint, $F^{\dagger}$ always exists, which may be unbounded (e.g. [6]).

Similar to the study of optimal feedback controls for LQ problems of SEEs, we introduce the following Riccati equations to characterize the optimal feedback operator:

$$
\left\{\begin{array}{l}
\dot{P}+P\left(A+A_{1}\right)+\left(A+A_{1}\right)^{*} P+C^{*} P C+Q-L^{*} K^{\dagger} L=0 \quad \text { in }[t, T), \\
P(T)=G
\end{array}\right.
$$

and

$$
\left\{\begin{array}{l}
\dot{\Pi}+\Pi\left(A+A_{1}+\widehat{A}\right)+\left(A+A_{1}+\widehat{A}\right)^{*} \Pi+Q+\widehat{Q}+(C+\widehat{C})^{*} P(C+\widehat{C})-\widehat{L}^{*} \widehat{K}^{\dagger} \widehat{L}=0 \quad \text { in }[t, T), \\
\Pi(T)=G+\widehat{G},
\end{array}\right.
$$

where

$$
\begin{array}{ll}
K \triangleq R+D^{*} P D, & \widehat{K} \triangleq R+\widehat{R}+(D+\widehat{D})^{*} P(D+\widehat{D}), \\
L \triangleq B^{*} P+D^{*} P C, & \widehat{L} \triangleq(B+\widehat{B})^{*} \Pi+(D+\widehat{D})^{*} P(C+\widehat{C}),
\end{array}
$$

and $K^{\dagger}\left(\right.$ resp. $\left.\widehat{K}^{\dagger}\right)$ denotes the generalized pseudo inverse of $K($ resp. $\widehat{K})$.

Definition 1.5. We call $P(\cdot) \in C_{\mathcal{S}}([t, T] ; \mathbb{S}(H))$ a regular solution to $(1.10)$ if

$$
\left\{\begin{array}{l}
K(s) \geq 0 \text { for a.e. } s \in[t, T] \\
\mathcal{R}(L(s)) \subseteq \mathcal{R}(K(s)) \text { for a.e. } s \in[t, T] \\
K^{\dagger} L \in L^{2}(t, T ; \mathcal{L}(H ; U))
\end{array}\right.
$$

and for any $\eta \in H$ and $s \in[t, T)$, it holds that

$$
P(s) \eta=e^{A^{*}(T-s)} G e^{A(T-s)} \eta+\int_{s}^{T} e^{A^{*}(\tau-s)}\left(P A_{1}+A_{1}^{*} P+C^{*} P C+Q-L^{*} K^{\dagger} L\right) e^{A(\tau-s)} \eta d \tau .
$$

Definition 1.6. We call $\Pi(\cdot) \in C_{\mathcal{S}}([t, T] ; \mathbb{S}(H))$ a regular solution to $(1.11)$ if

$$
\left\{\begin{array}{l}
\widehat{K}(s) \geq 0 \text { for a.e. } s \in[t, T] \\
\mathcal{R}(\widehat{L}(s)) \subseteq \mathcal{R}(\widehat{K}(s)) \text { for a.e. } s \in[t, T] \\
\widehat{K}^{\dagger} \widehat{L} \in L^{2}(t, T ; \mathcal{L}(H ; U))
\end{array}\right.
$$


and for any $\eta \in H$ and $s \in[t, T)$, it holds that

$$
\begin{aligned}
\Pi(s) \eta= & e^{A^{*}(T-s)}(G+\widehat{G}) e^{A(T-s)} \eta \\
& +\int_{s}^{T} e^{A^{*}(\tau-s)}\left[P\left(A_{1}+\widehat{A}\right)+\left(A_{1}+\widehat{A}\right)^{*} P+(C+\widehat{C})^{*} P(C+\widehat{C})+Q+\widehat{Q}-\widehat{L}^{*} \widehat{K}^{\dagger} \widehat{L}\right] e^{A(\tau-s)} \eta d \tau .
\end{aligned}
$$

Definition 1.7. The Riccati equation (1.10) (resp. (1.11)) is said to be regularly solvable on $[t, T]$ if it admits a regular solution.

Remark 1.8. The notion of regular solution, which is closely related to the existence of optimal feedback operators, is first introduced in [20] for LQ problems of SDEs.

The first main result in this paper is as follows.

Theorem 1.9. Problem (MF-SLQ) is closed-loop solvable on $[t, T]$ if and only if the Riccati equations (1.10) and (1.11) admit a regular solution $P(\cdot)$ and $\Pi(\cdot)$, respectively. Moreover, the optimal feedback operator $(\bar{\Theta}(\cdot), \widehat{\widehat{\Theta}}(\cdot))$ admits the following representation:

$$
\left\{\begin{array}{l}
\bar{\Theta}(\cdot)=-K(\cdot)^{\dagger} L(\cdot)+\left(I-K(\cdot)^{\dagger} K(\cdot)\right) \theta_{1}(\cdot), \\
\widehat{\widehat{\Theta}}(\cdot)=-\widehat{K}(\cdot)^{\dagger} \widehat{L}(\cdot)+\left(I-\widehat{K}(\cdot)^{\dagger} \widehat{K}(\cdot)\right) \theta_{2}(\cdot)-\bar{\Theta}(\cdot),
\end{array}\right.
$$

where $\theta_{1}(\cdot), \theta_{2}(\cdot) \in L^{2}(t, T ; \mathcal{L}(H ; U))$. Furthermore,

$$
\inf _{u(\cdot) \in \mathcal{U}[t, T]} \mathcal{J}(t, \xi ; u(\cdot))=\mathbb{E}\langle P(t)(\xi-\mathbb{E} \xi), \xi-\mathbb{E} \xi\rangle+\langle\Pi(t) \mathbb{E} \xi, \mathbb{E} \xi\rangle .
$$

Remark 1.10. Theorem 1.9 can be regarded as a nontrivial generalization of Theorem 2.1 in [16], where the author puts two additional assumptions, i.e., $A$ generates a $C_{0}$-group on $H$ and the eigenfunctions of $\mathrm{A}$ constitutes an orthonormal basis of $H$, to prove the result. Hence, the special case of Theorem 1.9 improves the main result in [16] noticeably.

Remark 1.11. Using Gronwall's inequality, one can show that (1.10) (resp. (1.11)) has at most one regular solution.

Definition 1.12. A regular solution $P(\cdot)$ to (1.10) is said to be strongly regular if

$$
K(s) \geq \lambda I \text { and } \widehat{K}(s) \geq \lambda I, \quad \text { a.e. } s \in[t, T],
$$

for some $\lambda>0$. If the Riccati equation (1.10) admits a strongly regular solution, it is said to be strongly regularly solvable.

Remark 1.13. It is clearly that if (1.10) is strongly regularly solvable, then (1.11) is regularly solvable.

Definition 1.14. The map $u(\cdot) \mapsto \mathcal{J}(t, 0 ; u(\cdot))$ is said to be uniformly convex if there exists a constant $\lambda>0$ such that

$$
\mathcal{J}(t, 0 ; u(\cdot)) \geq \lambda \mathbb{E} \int_{t}^{T}|u(s)|_{U}^{2} \mathrm{~d} s, \quad \forall u(\cdot) \in \mathcal{U}[t, T]
$$

We continue to present the second main result of this paper, which gives a relationship between the strongly regular solvability of the Riccati equation (1.10) and the uniform convexity of the cost functional. 
Theorem 1.15. The map $u(\cdot) \mapsto \mathcal{J}(t, 0 ; u(\cdot))$ is uniformly convex if and only if the Riccati equation (1.10) is strongly regularly solvable. In such case, the unique optimal control $\bar{u}(\cdot)$ of Problem (MF-SLQ) is

$$
\bar{u}(\cdot)=\bar{\Theta}(\cdot) \bar{x}(\cdot)+\overline{\widehat{\Theta}}(\cdot) \mathbb{E} \bar{x}(\cdot),
$$

where

$$
\bar{\Theta}(\cdot)=-K(\cdot)^{-1} L(\cdot), \quad \overline{\widehat{\Theta}}(\cdot)=-\widehat{K}(\cdot)^{-1} \widehat{L}(\cdot)+K(\cdot)^{-1} L(\cdot),
$$

and $\bar{x}(\cdot)$ solves $(1.8)$ with $(\Theta(\cdot), \widehat{\Theta}(\cdot))$ replaced by $(\bar{\Theta}(\cdot), \bar{\Theta}(\cdot))$ given in $(1.19)$. Moreover,

$$
\inf _{u(\cdot) \in \mathcal{U}[t, T]} \mathcal{J}(t, \xi ; u(\cdot))=\mathbb{E}\langle P(t)(\xi-\mathbb{E} \xi), \xi-\mathbb{E} \xi\rangle+\langle\Pi(t) \mathbb{E} \xi, \mathbb{E} \xi\rangle .
$$

The rest of the paper is organized as follows. In Section 2, we give some preliminaries. In Section 3, we provide a proof of Theorem 1.1 for the sake of completeness. Sections 4 and 5 are devoted to the proofs of Theorems 1.9 and 1.15 , respectively.

Since the operator-valued function $A_{1}$ does not lead to any difficulty for proofs of the main results, we assume that it equals zero for the simplicity of notations.

\section{SOME PRELIMINARY RESULTS}

In this section, we present some results to be used in the proofs of Theorems 1.9 and 1.15 .

\subsection{Some estimates for the control and the state}

Lemma 2.1. For any $u(\cdot) \in \mathcal{U}[t, T]$, let $x(\cdot ; t, 0, u)$ be the solution to (1.1) with the control $u(\cdot)$ and initial datum $\xi=0$. Then for any $(\Theta(\cdot), \widehat{\Theta}(\cdot)) \in \mathscr{F}[t, T]$, there exists a constant $\gamma>0$ such that

$$
\mathbb{E} \int_{t}^{T}|u(s)-\Theta[x(s ; t, 0, u)-\mathbb{E} x(s ; t, 0, u)]|_{U}^{2} \mathrm{~d} s \geq \gamma \mathbb{E} \int_{t}^{T}|u(s)|_{U}^{2} \mathrm{~d} s, \quad \forall u(\cdot) \in \mathcal{U}[t, T],
$$

and that

$$
\int_{t}^{T}|\mathbb{E} u(s)-(\Theta+\widehat{\Theta}) \mathbb{E} x(s ; t, 0, u)|_{U}^{2} \mathrm{~d} s \geq \gamma \int_{t}^{T}|\mathbb{E} u(s)|_{U}^{2} \mathrm{~d} s, \quad \forall u(\cdot) \in \mathcal{U}[t, T]
$$

Proof. We only prove (2.1). The proof for $(2.2)$ is analogous and simpler. Let $\Theta(\cdot) \in L^{2}(t, T ; \mathcal{L}(H ; U))$. Define an operator $\mathcal{A}: \mathcal{U}[t, T] \rightarrow \mathcal{U}[t, T]$ by

$$
(\mathcal{A} u)(\cdot)=u(\cdot)-\Theta[x(\cdot ; t, 0, u)-\mathbb{E} x(\cdot ; t, 0, u)] .
$$

Following the well-posedness of (1.1), $\mathcal{A}$ is linear and bounded.

Consider the following equation:

$$
\left\{\begin{aligned}
\mathrm{d} \widetilde{x}= & {[(A+B \Theta) \widetilde{x}+(\widehat{A}-B \Theta) \mathbb{E} \widetilde{x}+B v+\widehat{B} \mathbb{E} v] \mathrm{d} s } \\
& +[(C+D \Theta) \widetilde{x}+(\widehat{C}-\widehat{D} \Theta) \mathbb{E} \widetilde{x}+D v+\widehat{D} \mathbb{E} v] \mathrm{d} W(s) \quad \text { in }(t, T], \\
\widetilde{x}(t)= & 0 .
\end{aligned}\right.
$$


Define an operator $\mathcal{B}: \mathcal{U}[t, T] \rightarrow \mathcal{U}[t, T]$ as follows:

$$
(\mathcal{B} v)(\cdot)=v(\cdot)+\Theta[\tilde{x}(\cdot ; t, 0, v)-\mathbb{E} \tilde{x}(\cdot ; t, 0, v)] .
$$

By the well-posedness of $(2.3), \mathcal{B}$ is a bounded linear operator.

Taking $v=\mathcal{A} u$, then the equation (2.3) becomes

$$
\left\{\begin{aligned}
& \mathrm{d} \widetilde{x}=\{(A+B \Theta) \widetilde{x}+(\widehat{A}-B \Theta) \mathbb{E} \widetilde{x}+B[u-\Theta[x(s ; t, 0, u)-\mathbb{E} x(s ; t, 0, u)]]+\widehat{B} u\} \mathrm{d} s \\
&+\{(C+D \Theta) \widetilde{x}+(\widehat{C}-\widehat{D} \Theta) \mathbb{E} \widetilde{x}+D[u-\Theta[x(s ; t, 0, u)-\mathbb{E} x(s ; t, 0, u)]+\widehat{D} u\} \mathrm{d} W(s) \text { in }(t, T], \\
& \widetilde{x}(t)= 0
\end{aligned}\right.
$$

Clearly, $x(\cdot ; t, 0, u)$ solves the equation (2.4). By the uniqueness of the solution to (2.4), we know that $\widetilde{x}(\cdot ; t, 0, \mathcal{A} u)=x(\cdot ; t, 0, u)$. Therefore,

$$
(\mathcal{B A} u)(\cdot)=u(\cdot)-\Theta[x(\cdot ; t, 0, u)-\mathbb{E} x(\cdot ; t, 0, u)]+\Theta[x(\cdot ; t, 0, u)-\mathbb{E} x(\cdot ; t, 0, u)]=u(\cdot)
$$

Similarly, we can prove that $\mathcal{A B} u=u$. Thus, $\mathcal{A}$ is bijective and $\mathcal{B}$ is the inverse. Consequently,

$$
\begin{aligned}
\mathbb{E} \int_{t}^{T}|u(s)|_{U}^{2} \mathrm{~d} s & =\mathbb{E} \int_{t}^{T}\left|\left(\mathcal{A}^{-1} \mathcal{A} u\right)(s)\right|_{U}^{2} \mathrm{~d} s \leq\left|\mathcal{A}^{-1}\right|_{\mathcal{L}(\mathcal{U}[t, T])} \mathbb{E} \int_{t}^{T}|(\mathcal{A} u)(s)|_{U}^{2} \mathrm{~d} s \\
& =\left|\mathcal{A}^{-1}\right|_{\mathcal{L}(\mathcal{U}[t, T])} \mathbb{E} \int_{t}^{T}|u(s)-\Theta(s)[x(s ; t, 0, u)-\mathbb{E} x(s ; t, 0, u)]|_{U}^{2} \mathrm{~d} s, \quad \forall u(\cdot) \in \mathcal{U}[t, T] .
\end{aligned}
$$

This implies the inequality (2.1) with $\gamma=\left|\mathcal{A}^{-1}\right|_{\mathcal{L}(\mathcal{U}[t, T])}^{-1}$.

\subsection{An auxillary stochastic LQ problem}

In this subsection, we introduce a stochastic LQ problem, which is crucial in the proof of Theorem 1.15:

$$
\left\{\begin{array}{l}
\mathrm{d} \tilde{x}=(A \tilde{x}+B \tilde{u}) \mathrm{d} s+(C \tilde{x}+D \tilde{u}) \mathrm{d} W(s) \quad \text { in }(t, T] \\
\tilde{x}(t)=\tilde{\xi}
\end{array}\right.
$$

where $\tilde{\xi} \in L_{\mathcal{F}_{t}}^{2}(\Omega ; H)$ and $\tilde{u} \in \mathcal{U}[t, T]$. In $(2.5), \tilde{u}(\cdot)$ is the control and $\tilde{x}(\cdot)=\tilde{x}(\cdot ; t, \xi, u)$ is the state.

By the classical well-posedness result for stochastic evolution equations (e.g. [10], Chap. 6), for any $(t, \tilde{\xi}) \in$ $[0, T) \times L_{\mathcal{F}_{t}}^{2}(\Omega ; H)$ and any admissible control $\tilde{u}(\cdot) \in \mathcal{U}[t, T]$, the system (2.5) admits a unique mild solution $\tilde{x}(\cdot) \equiv \tilde{x}(\cdot ; t, \xi, \tilde{u}(\cdot)) \in C_{\mathbb{F}}\left([t, T] ; L^{2}(\Omega ; H)\right)$. Hence, the following cost functional is well-defined:

$$
\widetilde{\mathcal{J}}(t, \xi ; u(\cdot)) \triangleq \mathbb{E}\left[\langle G \tilde{x}(T), \tilde{x}(T)\rangle+\int_{t}^{T}(\langle Q \tilde{x}, \tilde{x}\rangle+\langle R \tilde{u}, \tilde{u}\rangle) \mathrm{d} s\right]
$$

Letting $\Theta \in L^{2}(0, T ; \mathcal{L}(H ; U))$, consider the following equation:

$$
\left\{\begin{array}{l}
\dot{P}_{\Theta}+P_{\Theta}(A+B \Theta)+(A+B \Theta)^{*} P_{\Theta}+(C+D \Theta)^{*} P_{\Theta}(C+D \Theta)+\Theta^{*} R \Theta+Q=0 \quad \text { in }[0, T), \\
P_{\Theta}(T)=G .
\end{array}\right.
$$


Recall that $P_{\Theta} \in C_{\mathcal{S}}([0, T] ; \mathbb{S}(H))$ is a mild solution to $(2.7)$ if for any $s \in[0, T]$,

$$
\begin{aligned}
P_{\Theta}(s) \eta= & e^{(T-s) A^{*}} G e^{(T-s) A} \eta+\int_{s}^{T} e^{(\tau-s) A^{*}}\left[P_{\Theta}\left(A_{1}+B \Theta\right)+\left(A_{1}+B \Theta\right)^{*} P_{\Theta}\right. \\
& \left.+(C+D \Theta)^{*} P_{\Theta}(C+D \Theta)+\Theta^{*} R \Theta+Q\right] e^{(\tau-s) A} \eta d \tau, \quad \forall \eta \in H .
\end{aligned}
$$

Let

$$
K_{\Theta}(\cdot) \triangleq R(\cdot)+D(\cdot)^{*} P_{\Theta}(\cdot) D(\cdot), \quad L_{\Theta}(\cdot)=B(\cdot)^{*} P_{\Theta}(\cdot)+D(\cdot)^{*} P_{\Theta}(\cdot) C(\cdot)
$$

The following lemma gives a relation between the cost functional and the equation (2.7).

Lemma 2.2. [16, Lemma 3.5] Let $P_{\Theta}(\cdot) \in C_{\mathcal{S}}([0, T] ; \mathbb{S}(H))$ be the mild solution of $(2.7)$. Then for any $(t, \tilde{\xi}) \in$ $[0, T) \times H$ and $\tilde{u}(\cdot) \in \mathcal{U}[t, T]$, we have

$$
\tilde{\mathcal{J}}(t, \tilde{\xi} ; \Theta(\cdot) \tilde{x}(\cdot)+\tilde{u}(\cdot))=\left\langle P_{\Theta}(t) \tilde{\xi}, \tilde{\xi}\right\rangle+\mathbb{E} \int_{t}^{T}\left[2\left\langle\left(L_{\Theta}+K_{\Theta} \Theta\right) \tilde{x}, \tilde{u}\right\rangle+\left\langle K_{\Theta} \tilde{u}, \tilde{u}\right\rangle\right] \mathrm{d} s
$$

From Lemma 2.2 and the well-posedness of (2.5), there exists $\alpha \in \mathbb{R}$, independent of $t$ and $\Theta$, such that

$$
\left\langle P_{\Theta}(t) \tilde{\xi}, \tilde{\xi}\right\rangle=\tilde{\mathcal{J}}(t, \tilde{\xi} ; 0) \geq \alpha|\tilde{\xi}|_{H}^{2}
$$

Next, we present a result for the positivity of $K_{\Theta}$.

Lemma 2.3. If the map $u \mapsto \mathcal{J}(t, 0 ; u(\cdot))$ is uniformly convex, then there exists $\lambda>0$ such that

$$
K_{\Theta}(s) \geq \lambda I, \quad \text { for a.e. } s \in[t, T] .
$$

Proof. Define

$$
\mathcal{U}_{0}[t, T] \triangleq\{v(\cdot) \in \mathcal{U}[t, T] \mid \mathbb{E} v(\cdot)=0\} .
$$

Let $\tilde{\xi}=0$ and $\tilde{u}(\cdot) \in \mathcal{U}_{0}[t, T]$. Then the corresponding solution $\tilde{x}(\cdot)$ satisfies that $\mathbb{E} \tilde{x}(\cdot)=0$. By the uniqueness of the solution to (1.1), $\tilde{x}(\cdot)$ also solves (1.1) with the control $u=\tilde{u}$. From (1.3) and (2.6) and noting that $\mathbb{E} \tilde{u}(\cdot)=0$ and $\mathbb{E} \tilde{x}(\cdot)=0$, we have $\mathcal{J}(t, 0 ; \tilde{u}(\cdot))=\tilde{\mathcal{J}}(t, 0 ; \tilde{u}(\cdot))$. Consequently, there exists $\lambda>0$ such that

$$
\tilde{\mathcal{J}}(t, 0 ; \tilde{u}(\cdot)) \geq \lambda \mathbb{E} \int_{t}^{T}|\tilde{u}(s)|_{U}^{2} \mathrm{~d} s, \quad \forall \tilde{u}(\cdot) \in \mathcal{U}_{0}[t, T] .
$$

This, together with Lemma 2.2, implies that

$$
\mathbb{E} \int_{t}^{T}\left[2\left\langle L_{\Theta} \tilde{x}, \tilde{u}\right\rangle+\left\langle K_{\Theta} \tilde{u}, \tilde{u}\right\rangle\right] \mathrm{d} s \geq \lambda \mathbb{E} \int_{t}^{T}|\tilde{u}(s)|_{U}^{2} \mathrm{~d} s, \quad \forall \tilde{u}(\cdot) \in \mathcal{U}_{0}[t, T]
$$

Let $\left\{u_{j}\right\}_{j=1}^{\infty}$ be dense in $U$. Denote by $\mathcal{T}_{j}$ the Lebesgue points of $\left\langle K_{\Theta} u_{j}, u_{j}\right\rangle$ in $[t, T]$. Then the Lebesgue measure of $\mathcal{T} \triangleq \bigcap_{j=1}^{\infty} \mathcal{T}_{j}$ is $T-t$. Let $s \in[t, T) \cap \mathcal{T}$ and take $\tilde{u}_{j}(\cdot)=W(t) u_{j} \chi_{[s, s+h]}(\cdot)$ with $s+h \leq T$. Then

$$
\left|\tilde{x}\left(\cdot ; t, \xi, \tilde{u}_{j}(\cdot)\right)\right|_{C_{\mathbb{F}}\left([t, T] ; L^{2}(\Omega ; H)\right)} \leq \mathcal{C}\left|\tilde{u}_{j}(\cdot)\right|_{L_{\mathbb{F}}^{2}(0, T ; U)} \leq \mathcal{C} \sqrt{t h}\left|u_{j}\right|_{U}
$$


From (2.12), we have

$$
\mathbb{E} \int_{s}^{s+h}\left[2\left\langle L_{\Theta} \tilde{x}\left(\cdot ; t, \xi, \tilde{u}_{j}(\cdot)\right), \tilde{u}_{j}\right\rangle+\left\langle K_{\Theta} \tilde{u}, \tilde{u}\right\rangle\right] \mathrm{d} s \geq \lambda \mathbb{E} \int_{s}^{s+h}\left|\tilde{u}_{j}(s)\right|_{U}^{2} \mathrm{~d} s, \quad \forall \tilde{u}(\cdot) \in \mathcal{U}_{0}[t, T] .
$$

Dividing both sides of (2.14) by $h$ and letting $h \rightarrow 0$, noting (2.13), we obtain

$$
\left\langle\left(K_{\Theta}(s)-\lambda I\right) u_{j}, u_{j}\right\rangle \geq 0, \quad \forall j \in \mathbb{N} .
$$

By the density of $\left\{u_{j}\right\}_{j=1}^{\infty}$, we know that

$$
K_{\Theta}(s) \geq \lambda I, \quad \text { for a.e. } s \in[t, T] .
$$

By (2.10) and Lemma 2.3, following the proof of Theorem 2.2 in [16] step by step, we can prove

Lemma 2.4. If the map $u(\cdot) \mapsto \mathcal{J}(t, 0 ; u(\cdot))$ is uniformly convex, then the equation (1.10) has a unique regular solution $P(\cdot)$ such that

$$
K(s)=R(s)+D(s)^{*} P(s) D(s) \geq \lambda I, \quad \text { for a.e. } s \in[t, T]
$$

where the $\lambda$ is the one in Lemma 2.3.

\subsection{An auxillary deterministic LQ problem}

In this subsection, as a preparation for the proof of Theorem 1.15, we introduce a deterministic LQ problem. Consider

$$
\left\{\begin{array}{l}
\frac{\mathrm{d} \check{x}}{\mathrm{~d} s}=(A+\widehat{A}) \check{x}+(B+\widehat{B}) v \quad \text { in }(t, T], \\
\check{x}(t)=\eta,
\end{array}\right.
$$

where $\eta \in H, v \in L^{2}(t, T ; U)$ is the control and $\check{x}(\cdot) \equiv \check{x}(\cdot ; t, \eta, v)$ is the state. Assume that the Riccati equation (1.10) has a regular solution $P$ such that (2.15) holds. The cost functional is

$$
\check{\mathcal{J}}(t, \eta ; v(\cdot)) \triangleq\langle(G+\widehat{G}) \check{x}(T), \check{x}(T)\rangle+\int_{t}^{T}(\langle\Upsilon \check{x}, \check{x}\rangle+2\langle\Gamma \check{x}, v\rangle+\langle\widehat{K} v, v\rangle) \mathrm{d} s,
$$

where

$$
\Upsilon=Q+\widehat{Q}+(C+\widehat{C})^{*} P(C+\widehat{C}), \quad \Gamma=(D+\widehat{D})^{*} P(C+\widehat{C}) .
$$

Consider the following deterministic LQ problem.

Problem (DLQ). For any given $(t, \eta) \in[0, T) \times H$, find a $\bar{v}(\cdot) \in L^{2}(t, T ; U)$, such that

$$
\check{\mathcal{J}}(t, \eta ; \bar{v}(\cdot))=\inf _{v(\cdot) \in L^{2}(t, T ; U)} \check{\mathcal{J}}(t, \eta ; v(\cdot)) .
$$

By the classical theory for LQ problems of deterministic evolution equations (e.g. [4], Part IV, Chap. 1, Sect. 2), we know that the Riccati equation associated with Problem (DLQ) is the equation (1.11). 
Lemma 2.5. If the map $v \mapsto \check{\mathcal{J}}(t, 0 ; v(\cdot))$ is uniformly convex, i.e., there exists $\lambda>0$ such that

$$
\check{\mathcal{J}}(t, 0 ; v(\cdot)) \geq \lambda \int_{t}^{T}|v(s)|^{2} \mathrm{~d} s, \quad \forall v(\cdot) \in L^{2}(t, T ; U),
$$

then the regular solution $P(\cdot)$ to (1.10) satisfies

$$
\widehat{K}(s) \geq \lambda I, \quad \text { for a.e. } s \in[t, T]
$$

and (1.11) admits a unique regular solution $\Pi(\cdot)$.

Proof. Once (2.20) holds, the well-posedness of (1.11) follows from the standard result for operator-valued equations (e.g. [4], Part IV, Chap. 1, Sect. 2). Hence, we only need to prove (2.20).

Assume the contrary. Then there exist $\delta>0$ and a measurable set $\mathfrak{T} \subset[t, T]$ such that its Lebesgue measure $\mathbf{m}(\mathfrak{T})>0$ and

$$
\widehat{K}(s)<(\lambda-\delta) I \quad \text { for } s \in \mathfrak{T}
$$

Let $N>0$ such that $\frac{1}{N} \leq \mathbf{m}(\mathfrak{T})$. Let $\left\{\mathfrak{T}_{n}\right\}_{n=1}^{\infty}$ be a sequence of measurable subsets of $\mathfrak{T}$ such that $\mathbf{m}\left(\mathfrak{T}_{n}\right)=\frac{1}{N+n}$. Let $h \in U$ and $v_{n}=n \chi_{\mathfrak{T}_{n}} h$ for $n=1,2, \cdots$ Denote by $\check{x}_{n}$ the solution to (2.16) with $\eta=0$ and $v=v_{n}$. Then we have

$$
\left|\check{x}_{n}\right|_{C([t, T] ; H)} \leq \mathcal{C}
$$

where $\mathcal{C}$ is a constant independent of $n$. Thus, by (2.17), we find that

$$
\begin{aligned}
& \varlimsup_{n \rightarrow \infty} \frac{1}{n} \check{\mathcal{J}}\left(t, 0 ; v_{n}(\cdot)\right) \\
& =\varlimsup_{n \rightarrow \infty} \frac{1}{n}\left[\left\langle(G+\widehat{G}) \check{x}_{n}(T), \check{x}_{n}(T)\right\rangle+\int_{t}^{T}\left(\left\langle\Upsilon \check{x}_{n}, \check{x}_{n}\right\rangle+2\left\langle\Gamma \check{x}_{n}, v_{n}\right\rangle+\left\langle\widehat{K} v_{n}, v_{n}\right\rangle\right) \mathrm{d} s\right] \\
& =\varlimsup_{n \rightarrow \infty} n \int_{\mathfrak{T}_{n}}\langle\widehat{K} h, h\rangle \mathrm{d} s \leq(\lambda-\delta)|h|_{U}^{2} .
\end{aligned}
$$

On the other hand, from (2.19), we see that

$$
\varliminf_{n \rightarrow \infty} \frac{1}{n} \check{\mathcal{J}}\left(t, 0 ; v_{n}(\cdot)\right) \geq \varliminf_{n \rightarrow \infty} \frac{1}{n} \int_{\mathfrak{T}_{n}}\left|v_{n}\right|_{U}^{2} \mathrm{~d} s \geq \lambda|h|_{U}^{2} .
$$

This contradicts (2.22). Consequently, (2.20) holds.

The next result concerns when the map $v(\cdot) \mapsto \check{\mathcal{J}}(t, 0 ; v(\cdot))$ is uniformly convex.

Lemma 2.6. If the map $u(\cdot) \mapsto \mathcal{J}(t, 0 ; u(\cdot))$ is uniformly convex, so does $v(\cdot) \mapsto \check{\mathcal{J}}(t, 0 ; v(\cdot))$.

Proof. Since the map $u(\cdot) \mapsto \mathcal{J}(t, 0 ; u(\cdot))$ is uniformly convex, by Lemma 2.4, the equation (1.10) has a unique regular solution $P(\cdot)$ such that $K(s) \geq \lambda I$, a.e. $s \in[t, T]$ for some $\lambda>0$. Put

$$
\Theta=-K^{-1} L=-\left(R+D^{*} P D\right)^{-1}\left(B^{*} P+D^{*} P C\right) \in L^{2}(t, T ; \mathcal{L}(H ; U)) .
$$


Then we have

$$
0=\dot{P}+P(A+B \Theta)+(A+B \Theta)^{*} P+(C+D \Theta)^{*} P(C+D \Theta)+\Theta^{*} R \Theta+Q
$$

Let $v(\cdot) \in L^{2}(t, T ; U)$. Let $x(\cdot)(\operatorname{resp} . \check{x}(\cdot))$ be the solution to $(1.1)(\operatorname{resp} .(2.16))$ where $u(\cdot)(\operatorname{resp} . v(\cdot))$ is replaced by $\Theta x(\cdot)+v(\cdot)(\operatorname{resp} . \Theta \check{x}(\cdot)+v(\cdot))$ and $\xi=0$, i.e., $x(\cdot)$ and $\check{x}(\cdot)$ are solutions to the following equations, respectively,

$$
\left\{\begin{aligned}
\mathrm{d} x= & {[A x+\widehat{A} \mathbb{E} x+B(\Theta x+v)+\widehat{B} \mathbb{E}(\Theta x+v)] \mathrm{d} s } \\
& +[C X+\widehat{C} \mathbb{E} x+D(\Theta x+v)+\widehat{D} \mathbb{E}(\Theta x+v)] \mathrm{d} W(s) \quad \text { in }(t, T], \\
x(t)= & 0
\end{aligned}\right.
$$

and

$$
\left\{\begin{array}{l}
\overline{\mathrm{d} s}=(A+\widehat{A}) \check{x}+(B+\widehat{B})(\Theta \check{x}+v) \quad \text { in }(t, T] \\
\check{x}(t)=0
\end{array}\right.
$$

Since $v(\cdot)$ is deterministic, from $(2.25)$, we have that $\mathbb{E} x(\cdot)$ solves $(2.26)$. By the uniqueness of the solution to $(2.26)$, we obtain that

$$
\mathbb{E} x(s)=\check{x}(s), \quad s \in[t, T] .
$$

Let $z(\cdot)=x(\cdot)-\mathbb{E} x(\cdot)$. Then $z(\cdot)$ solves

$$
\left\{\begin{array}{l}
\mathrm{d} z=(A+B \Theta) z \mathrm{~d} z+[(C+D \Theta) z+(C+\widehat{C}) \mathbb{E} x+(D+\widehat{D})(\Theta \mathbb{E} x+v)] \mathrm{d} W(s) \quad \text { in }(t, T] \\
z(t)=0
\end{array}\right.
$$

Denote by $\rho(A)$ the resolvent set of $A$. Let $l \in \rho(A)$ and $\mathbf{R}_{l}=l(l-A)^{-1}$. It is well known that for any $\eta \in H$, $\mathbf{R}_{l} \eta \in D(A)$ and

$$
\lim _{l \rightarrow \infty} \mathbf{R}_{l} \eta=\eta \quad \text { in } H
$$

Let $z_{l} \triangleq \mathbf{R}_{l} z$. Then it holds that (see [17], Lem. 2.7 for example)

$$
\lim _{l \rightarrow \infty} z_{l}=z \quad \text { in } C_{\mathbb{F}}\left([t, T] ; L^{2}(\Omega ; H)\right)
$$

and $z_{l}$ solves

$$
\left\{\begin{array}{l}
\mathrm{d} z_{l}=\left(A z_{l}+\mathbf{R}_{l} B \Theta z\right) \mathrm{d} s+\mathbf{R}_{l}[(C+D \Theta) z+(C+\widehat{C}) \mathbb{E} x+(D+\widehat{D})(\Theta \mathbb{E} x+v)] \mathrm{d} W(s) \text { in }(t, T] \\
z_{l}(t)=0
\end{array}\right.
$$


Applying Itô's formula to $\left\langle P(\cdot) z_{l}(\cdot), z_{l}(\cdot)\right\rangle$ and using (2.24), we have

$$
\begin{aligned}
& \mathbb{E}\left\langle G z_{l}(T), z_{l}(T)\right\rangle \\
= & -\mathbb{E} \int_{t}^{T}\left\langle\left[P(A+B \Theta)+(A+B \Theta)^{*} P+(C+D \Theta)^{*} P(C+D \Theta)+\Theta^{*} R \Theta+Q\right] z_{l}, z_{l}\right\rangle \mathrm{d} s \\
& +\mathbb{E} \int_{t}^{T}\left\langle P\left(A z_{l}+\mathbf{R}_{l} B \Theta z\right), z_{l}\right\rangle \mathrm{d} s+\mathbb{E} \int_{t}^{T}\left\langle P z_{l},\left(A z_{l}+\mathbf{R}_{l} B \Theta z\right)\right\rangle \mathrm{d} s \\
& +\mathbb{E} \int_{t}^{T}\left\langle P \mathbf{R}_{l}[(C+D \Theta) z+(C+\widehat{C}) \mathbb{E} x+(D+\widehat{D})(\Theta \mathbb{E} x+v)],\right. \\
& \left.\times \mathbf{R}_{l}[(C+D \Theta) z+(C+\widehat{C}) \mathbb{E} x+(D+\widehat{D})(\Theta \mathbb{E} x+v)]\right\rangle \mathrm{d} s \\
= & -\mathbb{E} \int_{t}^{T}\left\langle\left[P B \Theta+\Theta^{*} B^{*} P+(C+D \Theta)^{*} P(C+D \Theta)+\Theta^{*} R \Theta+Q\right] z_{l}, z_{l}\right\rangle \mathrm{d} s \\
& +\mathbb{E} \int_{t}^{T}\left\langle P \mathbf{R}_{l} B \Theta z, z_{l}\right\rangle \mathrm{d} s+\mathbb{E} \int_{t}^{T}\left\langle P z_{l}, \mathbf{R}_{l} B \Theta z\right\rangle \mathrm{d} s \\
& +\mathbb{E} \int_{t}^{T}\left\langle P \mathbf{R}_{l}[(C+D \Theta) z+(C+\widehat{C}) \mathbb{E} x+(D+\widehat{D})(\Theta \mathbb{E} x+v)],\right. \\
& \left.\times \mathbf{R}_{l}[(C+D \Theta) z+(C+\widehat{C}) \mathbb{E} x+(D+\widehat{D})(\Theta \mathbb{E} x+v)]\right\rangle \mathrm{d} s .
\end{aligned}
$$

Letting $l \rightarrow \infty$, by (2.29) and (2.30), we get that

$$
\begin{aligned}
& \mathbb{E}\langle G z(T), z(T)\rangle \\
= & -\mathbb{E} \int_{t}^{T}\left\langle\left[P B \Theta+\Theta^{*} B^{*} P+(C+D \Theta)^{*} P(C+D \Theta)+\Theta^{*} R \Theta+Q\right] z, z\right\rangle \mathrm{d} s \\
& +\mathbb{E} \int_{t}^{T}\langle P B \Theta z, z\rangle \mathrm{d} s+\mathbb{E} \int_{t}^{T}\langle P z, B \Theta z\rangle \mathrm{d} s \\
& +\mathbb{E} \int_{t}^{T}\langle P[(C+D \Theta) z+(C+\widehat{C}) \mathbb{E} x+(D+\widehat{D})(\Theta \mathbb{E} x+v)], \\
& \times[(C+D \Theta) z+(C+\widehat{C}) \mathbb{E} x+(D+\widehat{D})(\Theta \mathbb{E} x+v)]\rangle \mathrm{d} s \\
= & -\mathbb{E} \int_{t}^{T}\left\langle\left(\Theta^{*} R \Theta+Q\right) z, z\right\rangle \mathrm{d} s+2 \mathbb{E} \int_{t}^{T}\langle P(C+D \Theta) z,[(C+\widehat{C}) \mathbb{E} x+(D+\widehat{D})(\Theta \mathbb{E} x+v)]\rangle \mathrm{d} s \\
& +\mathbb{E} \int_{t}^{T}\langle P[(C+\widehat{C}) \mathbb{E} x+(D+\widehat{D})(\Theta \mathbb{E} x+v)],[(C+\widehat{C}) \mathbb{E} x+(D+\widehat{D})(\Theta \mathbb{E} x+v)]\rangle \mathrm{d} s .
\end{aligned}
$$

This implies that

$$
\begin{aligned}
& \mathcal{J}(t, 0 ; \Theta(\cdot) x(\cdot)+v(\cdot)) \\
= & \mathbb{E}\left\{\langle G x(T), x(T)\rangle+\langle\widehat{G} \mathbb{E} x(T), \mathbb{E} x(T)\rangle+\int_{t}^{T}[\langle Q x, x\rangle+\langle\widehat{Q} \mathbb{E} x, \mathbb{E} x\rangle\right. \\
& +\langle R(\Theta x+v), \Theta x+v\rangle+\langle\widehat{R} \mathbb{E}(\Theta x+v), \mathbb{E}(\Theta x+v)\rangle] \mathrm{d} s\} \\
= & \mathbb{E}\left[\langle G z(T), z(T)\rangle+\int_{t}^{T}(\langle Q z, z\rangle+\langle R \Theta z, \Theta z\rangle) \mathrm{d} s\right]+\langle(G+\widehat{G}) y(T), y(T)\rangle
\end{aligned}
$$




$$
\begin{aligned}
& +\int_{t}^{T}[\langle(Q+\widehat{Q}) \mathbb{E} x, \mathbb{E} x\rangle+\langle(R+\widehat{R})(\Theta \mathbb{E} x+v), \Theta \mathbb{E} x+v\rangle] \mathrm{d} s \\
= & \int_{t}^{T}\langle P[(C+\widehat{C}) \mathbb{E} x+(D+\widehat{D})(\Theta \mathbb{E} x+v)],(C+\widehat{C}) \mathbb{E} x+(D+\widehat{D})(\Theta \mathbb{E} x+v)\rangle \mathrm{d} s \\
& +\langle(G+\widehat{G}) \mathbb{E} x(T), \mathbb{E} x(T)\rangle+\int_{t}^{T}[\langle(Q+\widehat{Q}) \mathbb{E} x, \mathbb{E} x\rangle+\langle(R+\widehat{R})(\Theta \mathbb{E} x+v), \Theta \mathbb{E} x+v\rangle] \mathrm{d} s \\
= & \langle(G+\widehat{G}) \mathbb{E} x(T), \mathbb{E} x(T)\rangle+\int_{t}^{T}\left\{\left\langle\left[Q+\widehat{Q}+(C+\widehat{C})^{*} P(C+\widehat{C})\right] \mathbb{E} x, \mathbb{E} x\right\rangle\right. \\
& +2\left\langle\left[(D+\widehat{D})^{*} P(C+\widehat{C})\right] \mathbb{E} x, \Theta \mathbb{E} x+v\right\rangle \\
& \left.+\left\langle\left[R+\widehat{R}+(D+\widehat{D})^{*} P(D+\widehat{D})\right](\Theta \mathbb{E} x+v), \Theta \mathbb{E} x+v\right\rangle\right\} \mathrm{d} s \\
= & \breve{\mathcal{J}}(t, 0 ; \Theta(\cdot) \mathbb{E} x(\cdot)+v(\cdot)) .
\end{aligned}
$$

Due to the uniform convexity of $u \mapsto \mathcal{J}(t, 0 ; u(\cdot))$ and Jensen's inequality, we get from (2.31) that

$$
\begin{aligned}
& \check{\mathcal{J}}(t, 0 ; \Theta \mathbb{E} x(\cdot)+v(\cdot)) \\
& =\mathcal{J}(t, 0 ; \Theta x(\cdot)+v(\cdot)) \\
& \geq \delta \mathbb{E} \int_{t}^{T}|\Theta x+v|_{U}^{2} \mathrm{~d} s \geq \delta \int_{t}^{T}|\mathbb{E}[\Theta x+v]|_{U}^{2} \mathrm{~d} s=\delta \int_{t}^{T}|\Theta \mathbb{E} x+v|_{U}^{2} \mathrm{~d} s, \quad \forall v \in L^{2}(t, T ; U) .
\end{aligned}
$$

This concludes the uniform convexity of $v(\cdot) \mapsto \check{\mathcal{J}}(t, 0 ; v(\cdot))$.

Remark 2.7. Since $z(\cdot)$ may not be $D(A)$-valued, in the proof of Lemma 2.6, we introduce a family $\left\{z_{l}(\cdot)\right\}_{l \in \rho(A)}$ of semimartingales to apply Itô's formula to $\left\langle P(\cdot) z_{l}(\cdot), z_{l}(\cdot)\right\rangle_{H}$. The cost of doing this is that the equality we obtain is for $z_{\lambda}(\cdot)$ rather than the one for $z(\cdot)$, say $(2.30)$ for example. Fortunately, this can be handled by the following fact:

- The operator $A$ does not appear in the first and the last four lines of (2.30). Thus, by (2.29), and letting $l \rightarrow \infty$, we obtain an equality concerning $z(\cdot)$ rather than $z_{l}(\cdot)$.

In the rest of this paper, for saving space, we simply apply Itô's formula to the processes like $\langle P(\cdot) z(\cdot), z(\cdot)\rangle$ and some computations may be formal. But the result for such computations can be proved rigorously by the above procedures.

\subsection{A necessary condition for the optimal feedback operator}

In this subsection, we give necessary conditions for the optimal feedback operator of Problem (MF-SLQ).

Let $(\bar{\Theta}, \bar{\Theta}) \in \mathscr{F}[t, T]$ be an optimal feedback operator of Problem (MF-SLQ). Consider the following Lyapunov equations:

$$
\left\{\begin{array}{l}
\dot{\mathbf{P}}+\mathbf{P}(A+B \bar{\Theta})+(A+B \bar{\Theta})^{*} \mathbf{P}+(C+D \bar{\Theta})^{*} \mathbf{P}(C+D \bar{\Theta})+Q+\bar{\Theta}^{*} R \bar{\Theta}=0 \quad \text { in }[t, T) \\
\mathbf{P}(T)=G
\end{array}\right.
$$


and

$$
\left\{\begin{array}{l}
\dot{\boldsymbol{\Pi}}+\boldsymbol{\Pi}[A+\widehat{A}+(B+\widehat{B})(\bar{\Theta}+\overline{\widehat{\Theta}})]+[A+\widehat{A}+(B+\widehat{B})(\bar{\Theta}+\overline{\widehat{\Theta}})]^{*} \boldsymbol{\Pi}+Q+\widehat{Q} \\
+[C+\widehat{C}+(D+\widehat{D})(\bar{\Theta}+\overline{\widehat{\Theta}})]^{*} \mathbf{P}[C+\widehat{C}+(D+\widehat{D})(\bar{\Theta}+\widehat{\Theta})]+(\bar{\Theta}+\overline{\widehat{\Theta}})^{*}(R+\widehat{R})(\bar{\Theta}+\overline{\widehat{\Theta}})=0 \quad \text { in }[t, T), \\
\boldsymbol{\Pi}(T)=G+\widehat{G}
\end{array}\right.
$$

Put

$$
\begin{array}{ll}
\mathbf{K} \triangleq R+D^{*} \mathbf{P} D, & \widehat{\mathbf{K}} \triangleq R+\widehat{R}+(D+\widehat{D})^{*} \mathbf{P}(D+\widehat{D}), \\
\mathbf{L} \triangleq B^{*} \mathbf{P}+D^{*} \mathbf{P} C, & \widehat{\mathbf{L}} \triangleq(B+\widehat{B})^{*} \mathbf{\Pi}+(D+\widehat{D})^{*} \mathbf{P}(C+\widehat{C}) .
\end{array}
$$

Lemma 2.8. Let $\mathbf{P}(\cdot)$ (resp. $\Pi(\cdot)$ ) be the mild solution to (2.32) (resp. (2.33)). Then for a.e. $s \in[t, T]$,

$$
\mathbf{K}(s) \geq 0, \quad \mathbf{K}(s) \bar{\Theta}(s)+\mathbf{L}(s)=0,
$$

and

$$
\widehat{\mathbf{K}}(s) \geq 0, \quad \widehat{\mathbf{K}}(s) \widehat{\widehat{\Theta}}(s)+\widehat{\mathbf{L}}(s)=0 .
$$

Before proving Lemma 2.8, we introduce a family of auxiliary optimal control problems. Let $r \in[t, T)$ and $\xi \in L_{\mathcal{F}_{r}}^{2}(\Omega ; H)$. Consider the following control system:

$$
\left\{\begin{array}{l}
\mathrm{d} x=(A x+\widehat{A} \mathbb{E} x+B u+\widehat{B} \mathbb{E} u) \mathrm{d} s+(C x+\widehat{C} \mathbb{E} x+D u+\widehat{D} \mathbb{E} u) \mathrm{d} W(s) \quad \text { in }(r, T] \\
x(r)=\xi
\end{array}\right.
$$

The cost functional for $(2.36)$ is

$$
\begin{aligned}
\mathcal{J}(r, \xi ; u(\cdot)) \triangleq & \mathbb{E}\left[\langle G x(T), x(T)\rangle+\int_{r}^{T}(\langle Q x, x\rangle+\langle R u, u\rangle) \mathrm{d} s\right] \\
& +\langle\widehat{G} \mathbb{E} x(T), \mathbb{E} x(T)\rangle+\int_{r}^{T}(\langle\widehat{Q} \mathbb{E} x, \mathbb{E} x\rangle+\langle\widehat{R} \mathbb{E} u, \mathbb{E} u\rangle) \mathrm{d} s
\end{aligned}
$$

Consider the following optimal control problem:

Problem (MF-SLQ-r). For any given $(r, \xi) \in[t, T) \times L_{\mathcal{F}_{r}}^{2}(\Omega ; H)$, find a control $\bar{u}(\cdot) \in \mathcal{U}[r, T]$ such that

$$
\mathcal{J}(r, \xi ; \bar{u}(\cdot))=\inf _{u(\cdot) \in \mathcal{U}[r, T]} \mathcal{J}(r, \xi ; u(\cdot)) .
$$

For any $(\Theta(\cdot), \widehat{\Theta}(\cdot)) \in \mathscr{F}[t, T]$ and $\xi \in L_{\mathcal{F}_{r}}^{2}(\Omega ; H)$, let $x(\cdot) \equiv x(\cdot ; r, \xi, \Theta(\cdot), \widehat{\Theta}(\cdot))$ be the solution of the following closed-loop system:

$$
\left\{\begin{aligned}
\mathrm{d} x= & \{(A+B \Theta) x+[\widehat{A}+B \widehat{\Theta}+\widehat{B}(\Theta+\widehat{\Theta})] \mathbb{E} x\} \mathrm{d} s \\
& +\{(C+D \Theta) x+[\widehat{C}+D \widehat{\Theta}+\widehat{D}(\Theta+\widehat{\Theta})] \mathbb{E} x\} \mathrm{d} W(s) \quad \text { in }(r, T], \\
x(r)= & \xi
\end{aligned}\right.
$$


Definition 2.9. A feedback operator $(\bar{\Theta}(\cdot), \widehat{\widehat{\Theta}}(\cdot)) \in \mathscr{C}[t, T]$ is optimal on $[r, T]$ if

$$
\mathcal{J}(r, \xi ; \bar{\Theta}(\cdot) \bar{x}(\cdot)+\overline{\widehat{\Theta}}(\cdot) \mathbb{E}[\bar{x}(\cdot)]) \leq \mathcal{J}(r, \xi ; u(\cdot)), \quad \forall \xi \in L_{\mathcal{F}_{r}}^{2}(\Omega ; H), \forall u \in \mathcal{U}[r, T]
$$

where $\bar{x}(\cdot)$ is the solution to $(2.39)$ with $(\Theta, \widehat{\Theta})$ replaced by $(\bar{\Theta}(\cdot), \overline{\widehat{\Theta}}(\cdot))$. If an optimal feedback operator (uniquely) exists on $[r, T]$, Problem (MF-SLQ-r) is said to be (uniquely) closed-loop solvable on $[r, T]$.

Clearly, if $(\bar{\Theta}(\cdot), \overline{\widehat{\Theta}}(\cdot)) \in \mathscr{C}[t, T]$ is an optimal feedback operator for Problem (MF-SLQ) on $[t, T]$, then it is also optimal for Problem (MF-SLQ-r) on $[r, T]$.

Proof of Lemma 2.8. We borrow some ideas from the proof of ([24], Thm. 3.1) and divide the proof into three steps.

Step 1. In this step, we derive an integral type necessary condition for $\mathbf{K}$ and $\mathbf{L}$.

For any $\xi \in L_{\mathcal{F}_{r}}^{2}(\Omega ; H)$ and $v(\cdot) \in L_{\mathbb{F}}^{2}(r, T ; U)$, the system $(2.36)$ with the control $u(\cdot)=\bar{\Theta}(\cdot) x(\cdot)+\widehat{\Theta}(\cdot) \mathbb{E} x(\cdot)+$ $v(\cdot)$ becomes

$$
\left\{\begin{aligned}
\mathrm{d} x=\{ & (A+B \bar{\Theta}) x+B v+[\widehat{A}+\widehat{B} \bar{\Theta}+(B+\widehat{B}) \widehat{\widehat{\Theta}}] \mathbb{E} x+\widehat{B} \mathbb{E} v\} \mathrm{d} s \\
& +\{(C+D \bar{\Theta}) x+D v+[\widehat{C}+\widehat{D} \bar{\Theta}+(D+\widehat{D}) \widehat{\widehat{\Theta}}] \mathbb{E} x+\widehat{D} \mathbb{E} v\} \mathrm{d} W(s) \quad \text { in }(r, T], \\
x(r)= & \xi
\end{aligned}\right.
$$

and $\mathbb{E} x(\cdot)$ satisfies

$$
\left\{\begin{array}{l}
d \mathbb{E} x=[A+\widehat{A}+(B+\widehat{B})(\bar{\Theta}+\overline{\widehat{\Theta}})] \mathbb{E} x \mathrm{~d} s+(B+\widehat{B}) v d s \quad \text { in }(r, T], \\
\mathbb{E}[x(r)]=\mathbb{E} \xi
\end{array}\right.
$$

Set

$$
\begin{gathered}
\mathcal{M}_{r} \triangleq\left\{\left.g \in L_{\mathcal{F}_{r}}^{2}(\Omega ; \mathbb{R})|| g\right|_{L_{\mathcal{F}_{r}}^{2}(\Omega ; \mathbb{R})}=1, \mathbb{E} g=0\right\}, \\
\mathcal{T}_{r} \triangleq\left\{g \in L_{\mathcal{F}_{r}}^{2}(\Omega ; H) \mid g=g_{1} g_{2}, g_{1} \in \mathcal{M}_{r}, g_{2} \in H\right\}
\end{gathered}
$$

and

$$
\mathcal{O}_{r} \triangleq\left\{f \in L_{\mathbb{F}}^{2}(r, T ; U) \mid f=f_{1} f_{2}, f_{1} \in \mathcal{M}_{r}, f_{2} \in L^{2}(r, T ; U)\right\}
$$

Choose $\xi \in \mathcal{T}_{r}$ and $v \in \mathcal{O}_{r}$. Then the solution $x(\cdot)$ to (2.41) fulfills $\mathbb{E} x=0$ and (2.41) becomes

$$
\left\{\begin{array}{l}
\mathrm{d} x=[(A+B \bar{\Theta}) x+B v] \mathrm{d} s+[(C+D \bar{\Theta}) x+D v] \mathrm{d} W(s) \quad \text { in }(r, T] \\
x(r)=\xi
\end{array}\right.
$$


By Itô's formula, we have

$$
\begin{aligned}
\mathbb{E}\langle G x(T), x(T)\rangle-\mathbb{E}\langle\mathbf{P}(r) \xi, \xi\rangle \\
=\mathbb{E} \int_{r}^{T}\{-\langle\mathbf{P} x,(A+B \bar{\Theta}) x\rangle-\langle\mathbf{P}(A+B \bar{\Theta}) x, x\rangle-\langle\mathbf{P}(C+D \bar{\Theta}) x,(C+D \bar{\Theta}) x\rangle \\
\quad-\langle R \bar{\Theta} x, \bar{\Theta} x\rangle-\langle Q x, x\rangle+\langle\mathbf{P}[(A+B \bar{\Theta}) x+B v], x\rangle+\langle[(A+B \bar{\Theta}) x+B v], P x\rangle \\
\quad+\langle\mathbf{P}[(C+D \bar{\Theta}) x+D v],(C+D \bar{\Theta}) x+D v\rangle\} \mathrm{d} s \\
=\mathbb{E} \int_{r}^{T}[-\langle R \bar{\Theta} x, \bar{\Theta} x\rangle-\langle Q x, x\rangle+2\langle\mathbf{P} B v, x\rangle+2\langle\mathbf{P}(C+D \bar{\Theta}) x, D v\rangle+\langle P D v, D v\rangle] \mathrm{d} s .
\end{aligned}
$$

It follows from (2.37) and (2.44) that

$$
\begin{aligned}
& 2 \mathcal{J}(r, \xi ; \bar{\Theta}(\cdot) x(\cdot)+\overline{\widehat{\Theta}}(\cdot) \mathbb{E} x(\cdot)+v(\cdot)) \\
& =\mathbb{E}\left\{\langle G x(T), x(T)\rangle+\int_{r}^{T}[\langle Q x, x\rangle+\langle R(\bar{\Theta} x+v), \bar{\Theta} x+v\rangle] \mathrm{d} s\right\} \\
& =\mathbb{E}\langle\mathbf{P}(r) \xi, \xi\rangle+\mathbb{E} \int_{r}^{T}\left[\langle R v, v\rangle+2\langle R \bar{\Theta} x, v\rangle+2\langle\mathbf{P} B v, x\rangle+2\langle\mathbf{P}(C+D \bar{\Theta}) x, D v\rangle+\left\langle D^{*} \mathbf{P} D v, v\right\rangle\right] \mathrm{d} s \\
& =\mathbb{E}\langle\mathbf{P}(r) \xi, \xi\rangle+\mathbb{E} \int_{r}^{T}[\langle\mathbf{K} v, v\rangle+\langle(\mathbf{K} \bar{\Theta}+\mathbf{L}) x, v\rangle] \mathrm{d} s .
\end{aligned}
$$

Since $(\bar{\Theta}, \overline{\widehat{\Theta}})$ is an optimal feedback operator, we have

$$
\mathcal{J}(r, \xi ; \bar{\Theta}(\cdot) x(\cdot)+\overline{\widehat{\Theta}}(\cdot) \mathbb{E} x(\cdot)+v(\cdot)) \geq \mathcal{J}(r, \xi ; \bar{\Theta}(\cdot) x(\cdot)+\overline{\widehat{\Theta}}(\cdot) \mathbb{E} x(\cdot))=\mathbb{E}\langle\mathbf{P}(r) \xi, \xi\rangle .
$$

This, together with (2.45), implies that

$$
\mathbb{E} \int_{r}^{T}[\langle\mathbf{K} v, v\rangle+\langle(\mathbf{K} \bar{\Theta}+\mathbf{L}) x, v\rangle] \mathrm{d} s \geq 0, \quad \forall \xi \in \mathcal{T}_{r}, \forall v \in \mathcal{O}_{r}
$$

Step 2. In this step, we derive an integral type necessary condition for $\widehat{\mathbf{K}}$ and $\widehat{\mathbf{L}}$. By Itô's formula, we have

$$
\begin{aligned}
& \mathbb{E}[\langle G x(T), x(T)\rangle-\langle\mathbf{P}(r) \xi, \xi\rangle] \\
= & \mathbb{E} \int_{r}^{T}\{-\langle\mathbf{P} x,(A+B \bar{\Theta}) x\rangle-\langle\mathbf{P}(A+B \bar{\Theta}) x, x\rangle-\langle\mathbf{P}(C+D \bar{\Theta}) x,(C+D \bar{\Theta}) x\rangle \\
& -\langle R \bar{\Theta} x, \bar{\Theta} x\rangle-\langle Q x, x\rangle+\langle\mathbf{P}\{(A+B \bar{\Theta}) x+[\widehat{A}+\widehat{B} \bar{\Theta}+(B+\widehat{B}) \overline{\widehat{\Theta}}] \mathbb{E} x+(B+\widehat{B}) v\}, x\rangle \\
& +\langle\mathbf{P} x,(A+B \bar{\Theta}) x+[\widehat{A}+\widehat{B} \bar{\Theta}+(B+\widehat{B}) \widehat{\widehat{\Theta}}] \mathbb{E} x+(B+\widehat{B}) v\rangle \\
& +\langle\mathbf{P}\{(C+D \bar{\Theta}) x+[\widehat{C}+\widehat{D} \bar{\Theta}+(D+\widehat{D}) \overline{\widehat{\Theta}}] \mathbb{E} x+(D+\widehat{D}) v\}, \\
(C+D \bar{\Theta}) x+[\widehat{C}+\widehat{D} \bar{\Theta}+(D+\widehat{D}) \widehat{\Theta}] \mathbb{E} x+(D+\widehat{D}) v\rangle\} \mathrm{d} s & \\
= & \mathbb{E} \int_{r}^{T}\{-\langle R \bar{\Theta} x, \bar{\Theta} x\rangle-\langle Q x, x\rangle+\langle\mathbf{P}\{[\widehat{A}+\widehat{B} \bar{\Theta}+(B+\widehat{B}) \overline{\widehat{\Theta}}] \mathbb{E} x+(B+\widehat{B}) v\}, x\rangle \\
& +\langle\mathbf{P} x,[\widehat{A}+\widehat{B} \bar{\Theta}+(B+\widehat{B}) \overline{\widehat{\Theta}}] \mathbb{E} x+(B+\widehat{B}) v\rangle
\end{aligned}
$$




$$
\begin{aligned}
& +\langle\mathbf{P}\{[\widehat{C}+\widehat{D} \bar{\Theta}+(D+\widehat{D}) \overline{\widehat{\Theta}}] \mathbb{E} x+(D+\widehat{D}) v\},(C+D \bar{\Theta}) x\rangle \\
& +\langle\mathbf{P}(C+D \bar{\Theta}) x,[\widehat{C}+\widehat{D} \bar{\Theta}+(D+\widehat{D}) \widehat{\widehat{\Theta}}] \mathbb{E} x+(D+\widehat{D}) v\rangle \\
& +\langle\mathbf{P}\{[\widehat{C}+\widehat{D} \bar{\Theta}+(D+\widehat{D}) \overline{\widehat{\Theta}}] \mathbb{E} x+(D+\widehat{D}) v\},[\widehat{C}+\widehat{D} \bar{\Theta}+(D+\widehat{D}) \overline{\widehat{\Theta}}] \mathbb{E} x+(D+\widehat{D}) v\rangle\} \mathrm{d} s .
\end{aligned}
$$

By Newton-Leibniz formula, we get that

$$
\begin{aligned}
& \langle G \mathbb{E} x(T), \mathbb{E} x(T)\rangle-\langle\mathbf{P}(r) \mathbb{E} x(r), \mathbb{E} x(r)\rangle \\
= & \int_{r}^{T}\left\{-\langle\mathbf{P}(A+B \bar{\Theta}) \mathbb{E} x, \mathbb{E} x\rangle-\left\langle(A+B \bar{\Theta})^{*} \mathbf{P} \mathbb{E} x, \mathbb{E} x\right\rangle-\left\langle(C+D \bar{\Theta})^{*} \mathbf{P}(C+D \bar{\Theta}) \mathbb{E} x, \mathbb{E} x\right\rangle\right. \\
& -\langle Q \mathbb{E} x, \mathbb{E} x\rangle-\left\langle\bar{\Theta}^{*} R \bar{\Theta} \mathbb{E} x, \mathbb{E} x\right\rangle+\langle\mathbf{P}[A+\widehat{A}+(B+\widehat{B})(\bar{\Theta}+\widehat{\widehat{\Theta}})] \mathbb{E} x+\mathbf{P}(B+\widehat{B}) v, \mathbb{E} x\rangle \\
& +\langle\mathbb{E} x, \mathbf{P}[A+\widehat{A}+(B+\widehat{B})(\bar{\Theta}+\widehat{\widehat{\Theta}})] \mathbb{E} x+\mathbf{P}(B+\widehat{B}) v\rangle\} \mathrm{d} s \\
= & \int_{r}^{T}\left\{-\left\langle(C+D \bar{\Theta})^{*} \mathbf{P}(C+D \bar{\Theta}) \mathbb{E} x, \mathbb{E} x\right\rangle-\langle Q \mathbb{E} x, \mathbb{E} x\rangle-\left\langle\bar{\Theta}^{*} R \bar{\Theta} \mathbb{E} x, \mathbb{E} x\right\rangle\right. \\
& +\langle\mathbf{P}[A+\widehat{A}+\widehat{B} \bar{\Theta}+(B+\widehat{B}) \overline{\widehat{\Theta}}] \mathbb{E} x+\mathbf{P}(B+\widehat{B}) v, \mathbb{E} x\rangle \\
& +\langle\mathbb{E} x, \mathbf{P}[A+\widehat{A}+\widehat{B} \bar{\Theta}+(B+\widehat{B}) \overline{\widehat{\Theta}}] \mathbb{E} x+\mathbf{P}(B+\widehat{B}) v\rangle\} \mathrm{d} s
\end{aligned}
$$

and that

$$
\begin{aligned}
& \langle(G+\widehat{G}) \mathbb{E}[x(T)], \mathbb{E}[x(T)]\rangle-\langle\mathbf{\Pi}(r) \mathbb{E}[x(r)], \mathbb{E}[x(r)]\rangle \\
= & \mathbb{E} \int_{r}^{T}\left\{-\langle\mathbf{\Pi}[A+\widehat{A}+(B+\widehat{B})(\bar{\Theta}+\overline{\widehat{\Theta}})] \mathbb{E} x, \mathbb{E} x\rangle-\left\langle[A+\widehat{A}+(B+\widehat{B})(\bar{\Theta}+\overline{\widehat{\Theta}})]^{*} \boldsymbol{\Pi} \mathbb{E} x, \mathbb{E} x\right\rangle\right. \\
& -\left\langle[C+\widehat{C}+(D+\widehat{D})(\bar{\Theta}+\overline{\widehat{\Theta}})]^{*} \mathbf{P}[C+\widehat{C}+(D+\widehat{D})(\bar{\Theta}+\overline{\widehat{\Theta}})] \mathbb{E} x, \mathbb{E} x\right\rangle \\
& -\langle(Q+\widehat{Q}) \mathbb{E} x, \mathbb{E} x\rangle-\left\langle(\bar{\Theta}+\widehat{\widehat{\Theta}})^{*}(R+\widehat{R})(\bar{\Theta}+\widehat{\Theta}) \mathbb{E} x, \mathbb{E} x\right\rangle \\
& +\langle\boldsymbol{\Pi}[A+\widehat{A}+(B+\widehat{B})(\bar{\Theta}+\overline{\widehat{\Theta}})] \mathbb{E} x, \mathbb{E} x\rangle+\langle\mathbf{\Pi}(B+\widehat{B}) v, \mathbb{E} x\rangle \\
& +\langle\boldsymbol{\Pi} \mathbb{E} x,[A+\widehat{A}+(B+\widehat{B})(\bar{\Theta}+\overline{\widehat{\Theta}})] \mathbb{E} x\rangle+\langle(B+\widehat{B}) v, \mathbf{\Pi} \mathbb{E} x\rangle\} \mathrm{d} s \\
= & \mathbb{E} \int_{r}^{T}\left\{-\left\langle[C+\widehat{C}+(D+\widehat{D})(\bar{\Theta}+\widehat{\widehat{\Theta}})]^{*} \mathbf{P}[C+\widehat{C}+(D+\widehat{D})(\bar{\Theta}+\widehat{\widehat{\Theta}})] \mathbb{E} x, \mathbb{E} x\right\rangle\right. \\
& \left.-\langle(Q+\widehat{Q}) \mathbb{E} x, \mathbb{E} x\rangle+\left\langle\overline{\widehat{\Theta}}^{*} \widehat{R} \overline{\widehat{\Theta}} \mathbb{E} x, \mathbb{E} x\right\rangle+\langle\mathbf{\Pi}(B+\widehat{B}) v, \mathbb{E} x\rangle+\langle(B+\widehat{B}) v, \mathbf{\Pi} \mathbb{E} x\rangle\right\} \mathrm{d} s .
\end{aligned}
$$

By (2.37), (2.47), (2.48) and (2.49), we have that

$$
\begin{aligned}
\mathcal{J} & (r, \xi ; \bar{\Theta}(\cdot) x(\cdot)+\overline{\widehat{\Theta}}(\cdot) \mathbb{E} x(\cdot)+v(\cdot)) \\
= & \mathbb{E}\{\langle G x(T), x(T)\rangle+\langle\widehat{G} \mathbb{E} x(T), \mathbb{E} x(T)\rangle \\
& +\int_{r}^{T}[\langle Q x, x\rangle+\langle R(\bar{\Theta} x+\overline{\widehat{\Theta}} \mathbb{E} x+v), \bar{\Theta} x+\overline{\widehat{\Theta}} \mathbb{E} x+v\rangle] \mathrm{d} s \\
& \left.+\int_{r}^{T}[\langle\widehat{Q} \mathbb{E} x, \mathbb{E} x\rangle+\langle\widehat{R} \mathbb{E}(\bar{\Theta} x+\overline{\widehat{\Theta}} \mathbb{E} x+v), \mathbb{E}(\bar{\Theta} x+\overline{\widehat{\Theta}} \mathbb{E} x+v)\rangle] \mathrm{d} s\right\} \\
= & \mathbb{E}\langle\mathbf{P}(r)(\xi-\mathbb{E} \xi), \xi-\mathbb{E} \xi\rangle+\langle\mathbf{\Pi}(r) \mathbb{E} \xi, \mathbb{E} \xi\rangle
\end{aligned}
$$




$$
\begin{aligned}
& +\mathbb{E} \int_{r}^{T}\{\langle R v, v\rangle+\langle\widehat{R} v, v\rangle+2\langle R(\bar{\Theta} x+\widehat{\widehat{\Theta}} \mathbb{E} x), v\rangle+2\langle\widehat{R}(\bar{\Theta} \mathbb{E} x+\widehat{\widehat{\Theta}} \mathbb{E} x), v\rangle \\
& +2\langle\mathbf{P}(D+\widehat{D}) v,(C+\widehat{C}) \mathbb{E} x\rangle+2\langle\mathbf{P}(D+\widehat{D}) \bar{\Theta} x,(D+\widehat{D}) v\rangle \\
& \left.+2\left\langle v,(D+\widehat{D})^{*} \mathbf{P}(D+\widehat{D}) \widehat{\widehat{\Theta}} \mathbb{E} x\right\rangle+\left\langle(D+\widehat{D})^{*} \mathbf{P}(D+\widehat{D}) v, v\right\rangle+2\langle\mathbf{\Pi}(B+\widehat{B}) v, \mathbb{E} x\rangle\right\} \mathrm{d} s \\
& =\mathbb{E}\langle\mathbf{P}(r)(\xi-\mathbb{E} \xi), \xi-\mathbb{E} \xi\rangle+\langle\mathbf{\Pi}(r) \mathbb{E} \xi, \mathbb{E} \xi\rangle+\mathbb{E} \int_{r}^{T}\{\langle\widehat{\mathbf{K}} v, v\rangle+\langle[\widehat{\mathbf{K}}(\bar{\Theta}+\widehat{\Theta})+\widehat{\mathbf{L}}] x, v\rangle\} \mathrm{d} s .
\end{aligned}
$$

Since $(\bar{\Theta}, \overline{\widehat{\Theta}})$ is an optimal feedback operator, we have

$$
\begin{aligned}
& \mathcal{J}(r, \xi ; \bar{\Theta}(\cdot) x(\cdot)+\overline{\widehat{\Theta}}(\cdot) \mathbb{E} x(\cdot)+v(\cdot)) \\
& \geq \mathcal{J}(r, \xi ; \bar{\Theta}(\cdot) x(\cdot)+\overline{\widehat{\Theta}}(\cdot) \mathbb{E} x(\cdot))=\mathbb{E}\langle\mathbf{P}(r)(\xi-\mathbb{E} \xi), \xi-\mathbb{E} \xi\rangle+\langle\mathbf{\Pi}(r) \mathbb{E} \xi, \mathbb{E} \xi\rangle .
\end{aligned}
$$

This, together with (2.50), implies that

$$
\mathbb{E} \int_{r}^{T}\{\langle\widehat{\mathbf{K}} v, v\rangle+\langle[\widehat{\mathbf{K}}(\bar{\Theta}+\overline{\widehat{\Theta}})+\widehat{\mathbf{L}}] x, v\rangle\} \mathrm{d} s \geq 0, \quad \forall \xi \in L_{\mathcal{F}_{r}}^{2}(\Omega ; H), \forall v \in L_{\mathbb{F}}^{2}(r, T ; U) .
$$

Step 3. In this step, we prove (2.34) and (2.35).

We first show that $\mathbf{K}(s) \geq 0$ for a.e. $s \in[t, T]$ by contradiction. Otherwise, there exist $\delta>0$ and a measurable set $\mathfrak{T} \subset[t, T]$ with Lebesgue measure $\mathbf{m}(\mathfrak{T})>0$ such that

$$
K(s)<-\delta I \quad \text { for a.e. } s \in \mathfrak{T} .
$$

Let $N>0$ such that $\frac{1}{N} \leq \mathbf{m}(\mathfrak{T})$. Let $\left\{\mathfrak{T}_{n}\right\}_{n=1}^{\infty}$ be a sequence of measurable subsets of $\mathfrak{T}$ such that $\mathbf{m}\left(\mathfrak{T}_{n}\right)=\frac{1}{N+n}$. Let $h \in \mathcal{M}_{r}, f \in U$ and $v_{n}=n \chi_{\mathfrak{T}_{n}} h f$ for $n=1,2, \cdots$ Denote by $x_{n}$ the solution of (2.43) with $\xi=0$ and $v=v_{n}$. Then we have

$$
\left|x_{n}\right|_{C_{\mathbb{F}}\left([t, T] ; L^{2}(\Omega ; H)\right)} \leq \mathcal{C},
$$

where $\mathcal{C}$ is a constant independent of $n$. This, together with (2.53), implies that

$$
\varliminf_{n \rightarrow \infty} \frac{1}{n^{2}} \int_{t}^{T}\left[\left\langle\mathbf{K} v_{n}, v_{n}\right\rangle+\left\langle(\mathbf{K} \bar{\Theta}+\mathbf{L}) x_{n}, v_{n}\right\rangle\right] \mathrm{d} s \leq-\delta|f|_{U}^{2} .
$$

On the other hand, by (2.46), we see that

$$
\varliminf_{n \rightarrow \infty} \frac{1}{n} \int_{t}^{T}\left[\left\langle\mathbf{K} v_{n}, v_{n}\right\rangle+\left\langle(\mathbf{K} \bar{\Theta}+\mathbf{L}) x_{n}, v_{n}\right\rangle\right] \mathrm{d} s \geq 0,
$$

which contradicts (2.54). As a result, we obtain that $\mathbf{K}(s) \geq 0$ for a.e. $s \in[t, T]$.

From (1.2) and noting that $\bar{\Theta} \in L^{2}(t, T ; \mathcal{L}(U ; H))$, there exist $M \in \mathbb{N}$ and $\left\{t_{\ell}\right\}_{\ell=0}^{M}$ with $r=t_{0}<t_{1}<\cdots<$ $t_{M}=T$, such that for any $\ell \in\{0, \cdots, M-1\}$,

$$
4 \sup _{s \in\left[t_{\ell}, t_{\ell+1}\right]}\left[\left(\int_{t_{\ell}}^{s}\left|e^{A(s-\alpha)} B \bar{\Theta}\right|_{\mathcal{L}(H)} \mathrm{d} \alpha\right)^{2}+\int_{r}^{s}\left|e^{A(s-\alpha)}(C+D \bar{\Theta})\right|_{\mathcal{L}(H)}^{2} \mathrm{~d} \alpha\right] \leq \frac{1}{2}
$$


Let $h \in \mathcal{M}_{r}, \xi=h \tilde{\xi}$ with $\tilde{\xi} \in H$ and $v_{k}=\frac{1}{k} h \tilde{v}$ with $k \in \mathbb{N}$ and $\tilde{v} \in L^{2}(t, T ; U)$. Denote by $x_{k}$ the solution to (2.43) with $v=v_{k}$. Denote by $x_{0}$ the solution to (2.43) with $v=0$. By (2.55), we have

$$
\begin{aligned}
& \sup _{s \in\left[r, t_{1}\right]} \mathbb{E}\left|x_{k}(s)-x_{0}(s)\right|_{H}^{2} \\
= & \sup _{s \in\left[r, t_{1}\right]} \mathbb{E} \mid \int_{r}^{s} e^{A(s-\alpha)} B \bar{\Theta}\left(x_{k}-x_{0}\right) \mathrm{d} \alpha+\int_{r}^{s} e^{A(s-\alpha)} B v_{k} \mathrm{~d} \alpha \\
& +\int_{r}^{s} e^{A(s-\alpha)}(C+D \bar{\Theta})\left(x_{k}-x_{0}\right) \mathrm{d} W(\alpha)+\left.\int_{r}^{s} e^{A(s-\alpha)} D v_{k} \mathrm{~d} W(\alpha)\right|_{H} ^{2} \\
\leq & 4 \sup _{s \in\left[r, t_{1}\right]}\left[\left(\int_{r}^{s}\left|e^{A(s-\alpha)} B \bar{\Theta}\right|_{\mathcal{L}(H)}\left|x_{k}-x_{0}\right|_{H} \mathrm{~d} \alpha\right)^{2}\right. \\
& \left.+\int_{t}^{s}\left|e^{A(s-\alpha)}(C+D \bar{\Theta})\right|_{\mathcal{L}(H)}^{2}\left|x_{k}-x_{0}\right|_{H}^{2} \mathrm{~d} \alpha\right]+\mathcal{C} \int_{r}^{t_{1}}\left|v_{k}\right|_{U}^{2} \mathrm{~d} \alpha \\
\leq & 4 \sup _{s \in\left[r, t_{1}\right]} \mathbb{E}\left|x_{k}(s)-x_{0}(s)\right|^{2} \sup _{s \in\left[r, t_{1}\right]}\left[\left(\int_{r}^{s}\left|e^{A(s-\alpha)} B \bar{\Theta}\right|_{\mathcal{L}(H)} \mathrm{d} \alpha\right)^{2}+\int_{r}^{s}\left|e^{A(s-\alpha)}(C+D \bar{\Theta})\right|_{\mathcal{L}(H)}^{2} \mathrm{~d} \alpha\right] \\
& +\mathcal{C} \int_{r}^{t_{1}}\left|v_{k}\right|_{U}^{2} \mathrm{~d} \alpha \\
\leq & \frac{1}{2} \sup _{s \in\left[r, t_{1}\right]} \mathbb{E}\left|x_{k}(s)-x_{0}(s)\right|^{2}+\mathcal{C} \int_{r}^{t_{1}}\left|v_{k}\right|_{U}^{2} \mathrm{~d} \alpha .
\end{aligned}
$$

This implies that

$$
\sup _{s \in\left[r, t_{1}\right]} \mathbb{E}\left|x_{k}(s)-x_{0}(s)\right|_{H}^{2} \leq \mathcal{C} \int_{r}^{t_{1}}\left|v_{k}\right|_{U}^{2} \mathrm{~d} \alpha
$$

Analogously, we can obtain that

$$
\sup _{s \in[r, T]} \mathbb{E}\left|x_{k}(s)-x_{0}(s)\right|_{H}^{2} \leq \mathcal{C} \int_{r}^{T}\left|v_{k}\right|_{U}^{2} \mathrm{~d} \alpha
$$

where the constant $\mathcal{C}$ is independent of $k$. Consequently,

$$
\lim _{k \rightarrow \infty} \sup _{s \in[r, T]} \mathbb{E}\left|x_{k}(s)-x_{0}(s)\right|_{H}^{2}=0
$$

It follows from (2.46) that

$$
\varliminf_{k \rightarrow \infty} k \mathbb{E} \int_{r}^{T}\left[\left\langle\mathbf{K} v_{k}, v_{k}\right\rangle+\left\langle(\mathbf{K} \bar{\Theta}+\mathbf{L}) x_{k}, v_{k}\right\rangle\right] \mathrm{d} s \geq 0
$$

This, together with (2.57) and the choice of $v_{k}$, implies that

$$
\mathbb{E} \int_{r}^{T}\left\langle(\mathbf{K} \bar{\Theta}+\mathbf{L}) x_{0}, h \tilde{v}\right\rangle \mathrm{d} s \geq 0, \quad \forall h \in \mathcal{M}_{r}, \quad \forall \tilde{\xi} \in H, \quad \forall \tilde{v} \in L^{2}(r, T ; U) .
$$


By the arbitrariness of $\tilde{v}$, we see that

$$
\mathbb{E} \int_{r}^{T}\left\langle(\mathbf{K} \bar{\Theta}+\mathbf{L}) x_{0}, h \tilde{v}\right\rangle \mathrm{d} s=0, \quad \forall h \in \mathcal{M}_{r}, \quad \forall \tilde{\xi} \in H, \quad \forall \tilde{v} \in L^{2}(r, T ; U) .
$$

Noting that the Brownian $W(\cdot)-W(r)$ on $[r, T]$ is independent of $\mathcal{F}_{r}$, we have that

$$
\begin{aligned}
\mathbb{E}\left(x_{0}(s) \mid \mathcal{F}_{r}\right) & =\mathbb{E}\left(e^{A(s-r)} h \tilde{\xi}+\int_{r}^{s} e^{A(s-\alpha)} B \bar{\Theta} x_{0} \mathrm{~d} \alpha+\int_{r}^{s} e^{A(s-\alpha)}(C+D \bar{\Theta}) x_{0} \mathrm{~d} W(\alpha) \mid \mathcal{F}_{r}\right) \\
& =e^{A(s-r)} h \tilde{\xi}+\int_{r}^{s} e^{A(s-\alpha)} B \bar{\Theta} \mathbb{E}\left(x_{0} \mid \mathcal{F}_{r}\right) \mathrm{d} \alpha .
\end{aligned}
$$

Thus, $\mathbb{E}\left(x_{0}(s) \mid \mathcal{F}_{r}\right)=h \tilde{x}^{r}(s)$, where $\tilde{x}^{r}(\cdot)$ solves

$$
\left\{\begin{array}{l}
\mathrm{d} \tilde{x}^{r}=(A+B \bar{\Theta}) \tilde{x}^{r} \mathrm{~d} s \quad \text { in }(r, T] \\
\tilde{x}^{r}(r)=\tilde{\xi}
\end{array}\right.
$$

Therefore, from (2.58), we obtain that for any $\tilde{\xi} \in H$ and $\tilde{v} \in L^{2}(r, T ; U)$,

$$
\mathbb{E} \int_{r}^{T}\left\langle(\mathbf{K} \bar{\Theta}+\mathbf{L}) x_{0}, h \tilde{v}\right\rangle \mathrm{d} s=\mathbb{E}\left(h^{2}\right) \int_{r}^{T}\left\langle(\mathbf{K} \bar{\Theta}+\mathbf{L}) \tilde{x}^{r}, \tilde{v}\right\rangle \mathrm{d} s=0 .
$$

Consequently,

$$
\int_{r}^{T}\left\langle(\mathbf{K} \bar{\Theta}+\mathbf{L}) \tilde{x}^{r}, \tilde{v}\right\rangle \mathrm{d} s=0, \quad \forall r \in[t, T], \forall \tilde{\xi} \in H, \forall \tilde{v} \in L^{2}(r, T ; U)
$$

Since $H$ and $U$ are separable, there are $\left\{\xi_{j}\right\}_{j=1}^{\infty} \subset D(A)$ and $\left\{\rho_{j}\right\}_{j=1}^{\infty} \subset U$, both dense in $H$ and $U$ respectively. By Lebesgue's differential theorem, for each $j, k \in \mathbb{N}$, there exists a subset $\mathcal{T}_{j k} \subset[t, T]$ with Lebesgue measure $\mathbf{m}\left(\mathcal{T}_{j k}\right)=T-t$ such that

$$
\lim _{\varepsilon \rightarrow 0} \frac{1}{\varepsilon} \int_{\tau}^{\tau+\varepsilon}\left\langle(\mathbf{K} \bar{\Theta}+\mathbf{L}) \xi_{j}, \rho_{k}\right\rangle \mathrm{d} s=\left\langle(\mathbf{K}(\tau) \bar{\Theta}(\tau)+\mathbf{L}(\tau)) \xi_{j}, \rho_{k}\right\rangle, \quad \forall \tau \in \mathcal{T}_{j k}
$$

and

$$
\lim _{\varepsilon \rightarrow 0} \frac{1}{\varepsilon} \int_{\tau}^{\tau+\varepsilon} B \bar{\Theta} \xi_{j} \mathrm{~d} s=B(\tau) \bar{\Theta}(\tau) \xi_{j}, \quad \forall \tau \in \mathcal{T}_{j k}
$$

Let $\tau \in \mathcal{T}_{j} \triangleq \bigcap_{k=1}^{\infty} \mathcal{T}_{j k}$ and $\tilde{x}_{j}^{\tau}$ be the solution to

$$
\left\{\begin{array}{l}
\mathrm{d} \tilde{x}_{j}^{\tau}=(A+B \bar{\Theta}) \tilde{x}_{j}^{\tau} \mathrm{d} s \quad \text { in }(\tau, T] \\
\tilde{x}_{j}^{\tau}(\tau)=\xi_{j}
\end{array}\right.
$$

Then

$$
\lim _{\varepsilon \rightarrow 0} \frac{\tilde{x}_{j}^{\tau}(\tau+\varepsilon)-\xi_{j}}{\varepsilon}=(A+B(\tau) \bar{\Theta}(\tau)) \xi_{j} \quad \text { in } H
$$


This implies that

$$
\begin{aligned}
& \lim _{\varepsilon \rightarrow 0} \frac{1}{\varepsilon}\left|\int_{\tau}^{\tau+\varepsilon}\left\langle(\mathbf{K} \bar{\Theta}+\mathbf{L})\left(\tilde{x}_{j}^{\tau}(s)-\xi_{j}\right), \rho_{k}\right\rangle \mathrm{d} s\right| \\
& \leq \lim _{\varepsilon \rightarrow 0}\left(\int_{\tau}^{\tau+\varepsilon}|(\mathbf{K} \bar{\Theta}+\mathbf{L})|_{\mathcal{L}(H)}^{2} \frac{\left|\left(\tilde{x}_{j}^{\tau}(s)-\xi_{j}\right)\right|_{H}^{2}}{\varepsilon} \mathrm{d} s\right)^{\frac{1}{2}}\left|\rho_{k}\right|_{U}=0 .
\end{aligned}
$$

Let $\tilde{v}=\chi_{[\tau, \tau+\varepsilon]} \rho_{k}$. From $(2.61)$, we have that

$$
\int_{\tau}^{\tau+\varepsilon}\left\langle(\mathbf{K} \bar{\Theta}+\mathbf{L}) \tilde{x}_{j}^{\tau}(s), \rho_{k}\right\rangle \mathrm{d} s=0
$$

This, together with (2.63) and (2.65), implies that for any $\tau \in \mathcal{T}_{j k}$,

$$
\begin{aligned}
0 & =\lim _{\varepsilon \rightarrow 0} \frac{1}{\varepsilon} \int_{\tau}^{\tau+\varepsilon}\left\langle(\mathbf{K} \bar{\Theta}+\mathbf{L}) \tilde{x}_{j}^{\tau}(s), \rho_{k}\right\rangle \mathrm{d} s \\
& =\lim _{\varepsilon \rightarrow 0} \frac{1}{\varepsilon} \int_{\tau}^{\tau+\varepsilon}\left\langle(\mathbf{K} \bar{\Theta}+\mathbf{L}) \xi_{j}, \rho_{k}\right\rangle \mathrm{d} s \\
& =\left\langle(\mathbf{K}(\tau) \bar{\Theta}(\tau)+\mathbf{L}(\tau)) \xi_{j}, \rho_{k}\right\rangle .
\end{aligned}
$$

Consequently, we find that for every $\tau \in \bigcap_{j, k=1}^{\infty} \mathcal{T}_{j k}$,

$$
\left\langle(\mathbf{K}(\tau) \bar{\Theta}(\tau)+\mathbf{L}(\tau)) \xi_{j}, \rho_{k}\right\rangle=0, \quad \forall j, k \in \mathbb{N} .
$$

Since $\left\{\xi_{j}\right\}_{j=1}^{\infty}$ and $\left\{\rho_{j}\right\}_{j=1}^{\infty}$ are dense in $H$ and $U$, respectively, we get that

$$
\mathbf{K}(\tau) \bar{\Theta}(\tau)+\mathbf{L}(\tau)=0, \quad \forall \tau \in \bigcap_{j, k=1}^{\infty} \mathcal{T}_{j k} .
$$

Since the Lebesgue measure of all $\mathcal{T}_{j k}$ is $T-t$, we have that

$$
\mathbf{K}(\tau) \bar{\Theta}(\tau)+\mathbf{L}(\tau)=0, \quad \text { for a.e. } \tau \in[t, T] .
$$

This concludes (2.34). Similarly, we can prove (2.35).

\section{Proof of Theorem 1.1}

Proof of Theorem 1.1. The "if" part. Let $\xi \in L_{\mathcal{F}_{t}}^{2}(\Omega ; H), \delta \in \mathbb{R}$ and $u(\cdot), v(\cdot) \in \mathcal{U}[t, T]$. Let $x_{1}=x(\cdot ; t, \xi, u(\cdot)+$ $\delta v(\cdot))$ and $x_{0}(\cdot)=x(\cdot ; t, 0, v(\cdot))$. It follows from the linearity of the control system $(1.1)$ that $x_{1}(\cdot)=x(\cdot)+\delta x_{0}(\cdot)$. Hence,

$$
\begin{aligned}
& \mathcal{J}(t, \xi ; u(\cdot)+\delta v(\cdot))-\mathcal{J}(t, \xi ; u(\cdot)) \\
= & \delta \mathbb{E}\left\{\left\langle G\left(2 x(T)+\delta x_{0}(T)\right), x_{0}(T)\right\rangle+\int_{t}^{T}\left[\left\langle Q\left(2 x+\delta x_{0}\right), 2 x+\delta x_{0}\right\rangle+\langle R(2 u+\delta v), 2 u+\delta v\rangle\right] \mathrm{d} s\right\} \\
+ & \delta\left\{\left\langle\widehat{G}\left(2 \mathbb{E} x(T)+\delta \mathbb{E}\left[x_{0}(T)\right]\right), \mathbb{E}\left[x_{0}(T)\right]\right\rangle\right. \\
+ & \left.\int_{t}^{T}\left[\left\langle\widehat{Q}\left(2 \mathbb{E} x+\delta \mathbb{E}\left[x_{0}\right]\right), \mathbb{E}\left[x_{0}\right]\right\rangle+\langle\widehat{R}(2 \mathbb{E} u+\delta \mathbb{E} v), \mathbb{E} v\rangle\right] \mathrm{d} s\right\}
\end{aligned}
$$




$$
\begin{aligned}
= & 2 \delta \mathbb{E}\left[\left\langle G x(T), x_{0}(T)\right\rangle+\int_{t}^{T}\left(\left\langle Q x, x_{0}\right\rangle+\langle R u, v\rangle\right) \mathrm{d} s\right] \\
& +\delta^{2} \mathbb{E}\left[\left\langle G x_{0}(T), x_{0}(T)\right\rangle+\int_{t}^{T}\left(\left\langle Q x_{0}, x_{0}\right\rangle+\langle R v, v\rangle\right) \mathrm{d} s\right] \\
& +2 \delta\left[\left\langle\widehat{G} \mathbb{E} x(T), \mathbb{E}\left[x_{0}(T)\right]\right\rangle+\int_{t}^{T}\left(\left\langle\widehat{Q} \mathbb{E} x, \mathbb{E}\left[x_{0}\right]\right\rangle+\langle\widehat{R} \mathbb{E} u, \mathbb{E} v\rangle\right) \mathrm{d} s\right] \\
& +\delta^{2}\left[\left\langle\widehat{G} \mathbb{E}\left[x_{0}(T)\right], \mathbb{E}\left[x_{0}(T)\right]\right\rangle+\int_{t}^{T}\left(\left\langle\widehat{Q} \mathbb{E}\left[x_{0}\right], \mathbb{E}\left[x_{0}\right]\right\rangle+\langle\widehat{R} \mathbb{E} v, \mathbb{E} v\rangle\right) \mathrm{d} s\right] \\
= & 2 \delta \mathbb{E}\left[\left\langle G x(T)+\widehat{G} \mathbb{E} x(T), x_{0}(T)\right\rangle+\int_{t}^{T}\left(\left\langle Q x+\widehat{Q} \mathbb{E} x, x_{0}\right\rangle+\langle R u+\widehat{R} \mathbb{E} u, v\rangle\right) \mathrm{d} s\right] \\
& +\delta^{2} \mathcal{J}(t, 0 ; v(\cdot)) .
\end{aligned}
$$

Applying Itô's formula to $\left\langle y(\cdot), x_{0}(\cdot)\right\rangle$, we have

$$
\begin{aligned}
& \mathbb{E}\left\langle G x(T)+\widehat{G} \mathbb{E} x(T), x_{0}(T)\right\rangle \\
= & \mathbb{E} \int_{t}^{T}\left(-\left\langle A^{*} y+\widehat{A}^{*} \mathbb{E} y+C^{*} Y+\widehat{C}^{*} \mathbb{E} Y+Q x+\widehat{Q} \mathbb{E} x, x_{0}\right\rangle\right. \\
& \left.+\left\langle A x_{0}+\widehat{A} \mathbb{E}\left[x_{0}\right]+B v+\widehat{B} \mathbb{E} v, y\right\rangle+\left\langle C x_{0}+\widehat{C} \mathbb{E}\left[x_{0}\right]+D v+\widehat{D} \mathbb{E} v, Y\right\rangle\right) \mathrm{d} s \\
= & \mathbb{E} \int_{t}^{T}\left(\left\langle B^{*} y+\widehat{B}^{*} \mathbb{E} y+D^{*} Y+\widehat{D}^{*} \mathbb{E} Y, v\right\rangle-\left\langle Q x+\widehat{Q} \mathbb{E} x, x_{0}\right\rangle\right) \mathrm{d} s .
\end{aligned}
$$

If follows from (3.1) and (3.2) that for any $v(\cdot) \in \mathcal{U}[t, T]$,

$$
\begin{aligned}
& \mathcal{J}(t, \xi ; u(\cdot)+\delta v(\cdot))-\mathcal{J}(t, \xi ; u(\cdot)) \\
& =\delta^{2} \mathcal{J}(t, 0 ; v(\cdot))+2 \delta \mathbb{E} \int_{t}^{T}\left\langle B^{*} y+\widehat{B}^{*} \mathbb{E} y+D^{*} Y+\widehat{D}^{*} \mathbb{E} Y+R u+\widehat{R} \mathbb{E} u, v\right\rangle \mathrm{d} s
\end{aligned}
$$

If (1.6) and (1.7) hold, then we get from (3.3) that

$$
\mathcal{J}(t, \xi ; u(\cdot)+\delta v(\cdot))-\mathcal{J}(t, \xi ; u(\cdot)) \geq 0, \quad \forall v(\cdot) \in \mathcal{U}[t, T] .
$$

Thus, $u$ is an optimal control.

The "only if" part. If $u$ is an optimal control, then we have (3.4). Dividing both sides of (3.3) by $\delta^{2}$ and letting $\delta$ tend to $\infty$, we find that (1.6) holds. Dividing both sides of (3.3) by $\delta$ and letting $\delta$ tend to zero, we get (1.7).

\section{Proof of Theorem 1.9}

Proof of Theorem 1.9. The "only if" part. Suppose that $(\bar{\Theta}(\cdot), \overline{\widehat{\Theta}}(\cdot))$ is an optimal feedback operator of Problem (MF-SLQ). Let $\mathbf{P}(\cdot)$ (resp. $\mathbf{\Pi}(\cdot)$ ) be the mild solution to (2.32) (resp. (2.33)). From (2.34), we find that

$$
\mathbf{K}(s) \geq 0 \text { for a.e. } s \in[t, T],
$$


and

$$
\mathcal{R}(\mathbf{L}(s)) \subseteq \mathcal{R}(\mathbf{K}(s)) \text { for a.e. } s \in[t, T] .
$$

Further, it holds that

$$
\mathbf{K}(s)^{\dagger} \mathbf{L}(s)=-\mathbf{K}(s)^{\dagger} \mathbf{K}(s) \bar{\Theta}(s) \text { for a.e. } s \in[t, T]
$$

Noting that $\mathbf{K}(s)^{\dagger} \mathbf{K}(s)$ is an orthogonal projection operator, we see that $\mathbf{K}^{\dagger} \mathbf{L} \in L^{2}(t, T ; \mathcal{L}(H ; U))$ and

$$
\bar{\Theta}=-\mathbf{K}^{\dagger} \mathbf{L}+\left(I-\mathbf{K}^{\dagger} \mathbf{K}\right) \theta_{1}
$$

for some $\theta_{1}(\cdot) \in L^{2}(t, T ; \mathcal{L}(H ; U))$. Consequently,

$$
\mathbf{L}^{*} \bar{\Theta}=\bar{\Theta}^{*} \mathbf{K} \mathbf{K}^{\dagger} \mathbf{L}=-\mathbf{L K}^{\dagger} \mathbf{L}
$$

This, together with (2.32), implies that for any $\eta \in H$ and $s \in[t, T)$,

$$
\mathbf{P}(s) \eta=e^{A^{*}(T-s)} G e^{A(T-s)} \eta+\int_{s}^{T} e^{A^{*}(\tau-s)}\left(C^{*} \mathbf{P} C+Q-\mathbf{L}^{*} \mathbf{K}^{\dagger} \mathbf{L}\right) e^{A(\tau-s)} \eta d \tau .
$$

Consequently, $\mathbf{P}$ is a regular solution to (1.10). Similarly, we can prove that $\boldsymbol{\Pi}$ is a regular solution to (1.11) and

$$
\overline{\widehat{\Theta}}=-\widehat{\mathbf{K}}^{\dagger} \widehat{\mathbf{L}}+\left(I-\widehat{\mathbf{K}}^{\dagger} \widehat{\mathbf{K}}\right) \theta_{2}-\bar{\Theta}
$$

where $\theta_{2}(\cdot) \in L^{2}(t, T ; \mathcal{L}(H ; U))$. Moreover, by $(2.51)$, we get

$$
\inf _{u(\cdot) \in \mathcal{U}[t, T]} \mathcal{J}(t, \xi ; u(\cdot))=\mathbb{E}\langle\mathbf{P}(t)(\xi-\mathbb{E} \xi), \xi-\mathbb{E} \xi\rangle+\langle\mathbf{\Pi}(t) \mathbb{E} \xi, \mathbb{E} \xi\rangle .
$$

The "if" part. Let $(\bar{\Theta}(\cdot), \overline{\widehat{\Theta}}(\cdot))$ be defined by (1.14). Then we have

$$
\left\{\begin{array}{l}
L=-K \bar{\Theta} \\
\widehat{L}=-\widehat{K}(\bar{\Theta}+\widehat{\widehat{\Theta}}) .
\end{array}\right.
$$

For any $\xi \in L_{\mathcal{F}_{t}}^{2}(\Omega ; H)$ and $u(\cdot) \in \mathcal{U}[t, T]$, let $x(\cdot) \equiv x(\cdot ; t, \xi, u(\cdot))$ be the corresponding solution to (1.1).

Applying Itô's formula to $\langle P(\cdot) x(\cdot), x(\cdot)\rangle$, we have

$$
\begin{aligned}
\mathbb{E} & \langle G x(T), x(T)\rangle-\mathbb{E}\langle P(t) \xi, \xi\rangle+\mathbb{E} \int_{t}^{T}(\langle Q x, x\rangle+\langle R u, u\rangle) \mathrm{d} s \\
= & \mathbb{E} \int_{t}^{T}\left[-\langle P A x, x\rangle-\langle P x, A x\rangle-\left\langle\left(C^{*} P C+Q-L^{*} K^{\dagger} L\right) x, x\right\rangle\right. \\
& +\langle P(A x+\widehat{A} \mathbb{E} x+B u+\widehat{B} \mathbb{E} u), x\rangle+\langle P x, A x+\widehat{A} \mathbb{E} x+B u+\widehat{B} \mathbb{E} u\rangle \\
& +\langle P(C x+\widehat{C} \mathbb{E} x+D u+\widehat{D} \mathbb{E} u), C x+\widehat{C} E x+D u+\widehat{D} \mathbb{E} u\rangle] \mathrm{d} s \\
& +\mathbb{E} \int_{t}^{T}(\langle Q x, x\rangle+\langle R u, u\rangle) \mathrm{d} s
\end{aligned}
$$




$$
\begin{aligned}
= & \mathbb{E} \int_{t}^{T}\left[\left\langle L^{*} K^{\dagger} L x, x\right\rangle+2\langle P x, \widehat{A} \mathbb{E} x+\widehat{B} \mathbb{E} u\rangle+2\left\langle\left(P B+C^{*} P D\right) u, x\right\rangle+\left\langle\left(R+D^{*} P D\right) u, u\right\rangle\right. \\
& +2\langle P(\widehat{C} \mathbb{E} x+\widehat{D} \mathbb{E} u), C x+D u\rangle+\langle P(\widehat{C} \mathbb{E} x+\widehat{D} \mathbb{E} u), \widehat{C} \mathbb{E} x+\widehat{D} \mathbb{E} u\rangle] \mathrm{d} s \\
= & \mathbb{E} \int_{t}^{T}\left[\left\langle\bar{\Theta}^{*} K \bar{\Theta} x, x\right\rangle-2\left\langle\bar{\Theta}^{*} K u, x\right\rangle+\langle K u, u\rangle+2\langle P x, \widehat{A} \mathbb{E} x+\widehat{B} \mathbb{E} u\rangle\right. \\
& +2\langle P(\widehat{C} \mathbb{E} x+\widehat{D} \mathbb{E} u), C x+D u\rangle+\langle P(\widehat{C} \mathbb{E} x+\widehat{D} \mathbb{E} u), \widehat{C} \mathbb{E} x+\widehat{D} \mathbb{E} u\rangle] \mathrm{d} s .
\end{aligned}
$$

Applying the integration by parts formula to $\langle P(\cdot) \mathbb{E} x(\cdot), \mathbb{E} x(\cdot)\rangle$, we have

$$
\begin{aligned}
& \mathbb{E}\langle G \mathbb{E} x(T), \mathbb{E} x(T)\rangle-\mathbb{E}\langle P(t) \mathbb{E} \xi, \mathbb{E} \xi\rangle+\mathbb{E} \int_{t}^{T}(\langle Q \mathbb{E} x, \mathbb{E} x\rangle+\langle R \mathbb{E} u, \mathbb{E} u\rangle) \mathrm{d} s \\
& =\mathbb{E} \int_{t}^{T}\left[-\langle P A \mathbb{E} x, \mathbb{E} x\rangle-\langle P \mathbb{E} x, A \mathbb{E} x\rangle-\left\langle\left(C^{*} P C+Q-L^{*} K^{\dagger} L\right) \mathbb{E} x, \mathbb{E} x\right\rangle\right. \\
& \quad+\langle P[A \mathbb{E} x+\widehat{A} \mathbb{E} x+(B+\widehat{B}) \mathbb{E} u], \mathbb{E} x\rangle+\langle P \mathbb{E} x, A \mathbb{E} x+\widehat{A} \mathbb{E} x+(B+\widehat{B}) \mathbb{E} u\rangle] \mathrm{d} s \\
& +\mathbb{E} \int_{t}^{T}(\langle Q \mathbb{E} x, \mathbb{E} x\rangle+\langle R \mathbb{E} u, \mathbb{E} u\rangle) \mathrm{d} s \\
& =\mathbb{E} \int_{t}^{T}\left[-\left\langle C^{*} P C \mathbb{E} x, \mathbb{E} x\right\rangle+\left\langle\bar{\Theta}^{*} K \bar{\Theta} \mathbb{E} x, \mathbb{E} x\right\rangle+2\langle P \mathbb{E} x, \widehat{A} \mathbb{E} x+(B+\widehat{B}) \mathbb{E} u\rangle+\langle R \mathbb{E} u, \mathbb{E} u\rangle\right] \mathrm{d} s .
\end{aligned}
$$

Applying the integration by parts formula to $\langle\Pi(\cdot) \mathbb{E} x(\cdot), \mathbb{E} x(\cdot)\rangle$, we obtain

$$
\begin{aligned}
\langle & (G+\widehat{G}) \mathbb{E} x(T), \mathbb{E} x(T)\rangle-\langle\Pi(t) \mathbb{E} \xi, \mathbb{E} \xi\rangle+\int_{t}^{T}(\langle(Q+\widehat{Q}) \mathbb{E} x, \mathbb{E} x\rangle+\langle(R+\widehat{R}) \mathbb{E} u, \mathbb{E} u\rangle) \mathrm{d} s \\
= & \int_{t}^{T}\left\{-\langle\Pi(A+\widehat{A}) \mathbb{E} x, \mathbb{E} x\rangle-\langle\Pi \mathbb{E} x,(A+\widehat{A}) \mathbb{E} x\rangle-\left\langle\left[Q+\widehat{Q}+(C+\widehat{C})^{*} P(C+\widehat{C})\right] \mathbb{E} x, \mathbb{E} x\right\rangle\right. \\
& \left.+\left\langle\widehat{L}^{*} \widehat{K^{\dagger}} \widehat{L} \mathbb{E} x, \mathbb{E} x\right\rangle+\langle\Pi[(A+\widehat{A}) \mathbb{E} x+(B+\widehat{B}) \mathbb{E} u], \mathbb{E} x\rangle+\langle\Pi \mathbb{E} x,(A+\widehat{A}) \mathbb{E} x+(B+\widehat{B}) \mathbb{E} u\rangle\right\} \mathrm{d} s \\
& +\int_{t}^{T}[\langle(Q+\widehat{Q}) \mathbb{E} x, \mathbb{E} x\rangle+\langle(R+\widehat{R}) \mathbb{E} u, \mathbb{E} u\rangle] \mathrm{d} s \\
= & \int_{t}^{T}\left\{\left\langle\left[-(C+\widehat{C})^{*} P(C+\widehat{C})+(\bar{\Theta}+\widehat{\Theta})^{*} \widehat{K}(\bar{\Theta}+\widehat{\Theta})\right] \mathbb{E} x, \mathbb{E} x\right\rangle+2\langle[\Pi(B+\widehat{B})] \mathbb{E} u, \mathbb{E} x\rangle\right. \\
& +\langle(R+\widehat{R}) \mathbb{E} u, \mathbb{E} u\rangle\} \mathrm{d} s .
\end{aligned}
$$

Combining (4.4), (4.5) and (4.6), we get that

$$
\begin{aligned}
& \mathcal{J}(t, \xi ; u(\cdot))-\mathbb{E}\langle P(t)(\xi-\mathbb{E} \xi), \xi-\mathbb{E} \xi\rangle-\langle\Pi(t) \mathbb{E} \xi, \mathbb{E} \xi\rangle \\
= & \mathbb{E} \int_{t}^{T}[\langle K \bar{\Theta} x, \bar{\Theta} x\rangle-2\langle K \bar{\Theta} x, u\rangle+\langle K u, u\rangle-\langle K \bar{\Theta} \mathbb{E} x, \bar{\Theta} \mathbb{E} x\rangle+2\langle K \bar{\Theta} \mathbb{E} x, \mathbb{E} u\rangle \\
& -\langle K \mathbb{E} x, \mathbb{E} x\rangle+\langle\widehat{K}(\bar{\Theta}+\overline{\widehat{\Theta}}) \mathbb{E} x,(\bar{\Theta}+\overline{\widehat{\Theta}}) \mathbb{E} x\rangle-2\langle\widehat{K}(\bar{\Theta}+\overline{\widehat{\Theta}}) \mathbb{E} x, \mathbb{E} u\rangle+\langle\widehat{K} \mathbb{E} u, \mathbb{E} u\rangle] \mathrm{d} s \\
= & \mathbb{E} \int_{t}^{T}[\langle K \bar{\Theta}(x-\mathbb{E} x), \bar{\Theta}(x-\mathbb{E} x)\rangle-2\langle K \bar{\Theta}(x-\mathbb{E} x), u-\mathbb{E} u\rangle+\langle K(u-\mathbb{E} u), u-\mathbb{E} u\rangle
\end{aligned}
$$




$$
\begin{aligned}
& +\langle\widehat{K}(\bar{\Theta}+\overline{\widehat{\Theta}}) \mathbb{E} x,(\bar{\Theta}+\overline{\widehat{\Theta}}) \mathbb{E} x\rangle-2\langle\widehat{K}(\bar{\Theta}+\overline{\widehat{\Theta}}) \mathbb{E} x, \mathbb{E} u\rangle+\langle\widehat{K} \mathbb{E} u, \mathbb{E} u\rangle] \mathrm{d} s \\
= & \mathbb{E} \int_{t}^{T}[\langle K[u-\mathbb{E} u-\bar{\Theta}(x-\mathbb{E} x)], u-\mathbb{E} u-\bar{\Theta}(x-\mathbb{E} x)\rangle \\
& +\langle\widehat{K}[\mathbb{E} u-(\bar{\Theta}+\overline{\widehat{\Theta}}) \mathbb{E} x], \mathbb{E} u-(\bar{\Theta}+\overline{\widehat{\Theta}}) \mathbb{E} x\rangle] \mathrm{d} s .
\end{aligned}
$$

Since $K, \widehat{K} \geq 0,(4.7)$ implies that

$$
\begin{aligned}
\mathcal{J}(t, \xi ; u(\cdot)) & \geq \mathbb{E}\langle P(t)(\xi-\mathbb{E} \xi), \xi-\mathbb{E} \xi\rangle+\langle\Pi(t) \mathbb{E} \xi, \mathbb{E} \xi\rangle \\
& =\mathcal{J}(t, \xi ; \bar{\Theta}(\cdot) \bar{x}(\cdot)+\widehat{\widehat{\Theta}}(\cdot) \mathbb{E}[\bar{x}(\cdot)]), \quad \forall(\xi, u(\cdot)) \in L_{\mathcal{F}_{t}}^{2}(\Omega ; H) \times \mathcal{U}[t, T]
\end{aligned}
$$

Therefore, $(\bar{\Theta}(\cdot), \overline{\widehat{\Theta}}(\cdot))$ is an optimal feedback operator of Problem (MF-SLQ) on $[t, T]$ and (1.15) holds. The proof is completed.

\section{Proof of Theorem 1.15}

Proof of Theorem 1.15. The "only if" part. By Lemmas 2.4, 2.6 and 2.5, the solution $P$ to (1.10) is strongly regular and (1.11) has a unique regular solution.

The "if" part. The proof is very similar to that of the "if" part of Theorem 1.9. For any $\xi \in L_{\mathcal{F}_{t}}^{2}(\Omega ; H)$ and $u(\cdot) \in \mathcal{U}[t, T]$, let $x(\cdot) \equiv x(\cdot ; t, \xi, u(\cdot))$ be the corresponding solution to (1.1).

Similar to the proof of $(4.7)$, we can obtain that

$$
\begin{aligned}
& \mathcal{J}(t, \xi ; u(\cdot))-\mathbb{E}\langle P(t)(\xi-\mathbb{E} \xi), \xi-\mathbb{E} \xi\rangle-\langle\Pi(t) \mathbb{E} \xi, \mathbb{E} \xi\rangle \\
& =\mathbb{E} \int_{t}^{T}[\langle K[u-\mathbb{E} u-\bar{\Theta}(x-\mathbb{E} x)], u-\mathbb{E} u-\bar{\Theta}(x-\mathbb{E} x)\rangle \\
& +\langle\widehat{K}[\mathbb{E} u-(\bar{\Theta}+\bar{\Theta}) \mathbb{E} x], \mathbb{E} u-(\bar{\Theta}+\overline{\widehat{\Theta}}) \mathbb{E} x\rangle] \mathrm{d} s .
\end{aligned}
$$

Since $K, \widehat{K} \geq \lambda I$ for some $\lambda>0,(5.1)$ implies that

$$
\mathcal{J}(t, \xi ; u(\cdot)) \geq \mathbb{E}\langle P(t)(\xi-\mathbb{E} \xi), \xi-\mathbb{E} \xi\rangle+\langle\Pi(t) \mathbb{E} \xi, \mathbb{E} \xi\rangle
$$

The equality holds if and only if

$$
u-\mathbb{E} u=\bar{\Theta}(x-\mathbb{E} x), \quad \mathbb{E} u=(\bar{\Theta}+\overline{\widehat{\Theta}}) \mathbb{E} x .
$$

This is equivalent to

$$
u=\bar{\Theta} x+\overline{\widehat{\Theta}} \mathbb{E} x .
$$


Take $\xi=0$. From (5.1), noting that $K, \widehat{K} \geq \lambda I$ for some $\lambda>0$ and using Lemma 2.1, we have

$$
\begin{aligned}
& \mathcal{J}(t, 0 ; u(\cdot)) \\
\geq & \lambda \mathbb{E} \int_{t}^{T}\left[|u-\mathbb{E} u-\bar{\Theta}(x-\mathbb{E} x)|_{U}^{2}+|\mathbb{E} u-(\bar{\Theta}+\overline{\widehat{\Theta}}) \mathbb{E} x|_{U}^{2}\right] \mathrm{d} s \\
\geq & \lambda \mathbb{E} \int_{t}^{T}\left[|u-\bar{\Theta}(x-\mathbb{E} x)|_{U}^{2}-2\langle u-\bar{\Theta}(x-\mathbb{E} x), \mathbb{E} u\rangle+(1+\gamma)|\mathbb{E} u|_{U}^{2}\right] \mathrm{d} s \\
\geq & \frac{\lambda \gamma}{1+\gamma} \mathbb{E} \int_{t}^{T}|u-\bar{\Theta}(x-\mathbb{E} x)|_{U}^{2} \mathrm{~d} s \geq \frac{\lambda \gamma^{2}}{1+\gamma} \mathbb{E} \int_{t}^{T}|u(s)|_{U}^{2} \mathrm{~d} s, \quad \forall u(\cdot) \in \mathcal{U}[t, T],
\end{aligned}
$$

for some $\gamma>0$. The uniform convexity of $u(\cdot) \mapsto \mathcal{J}(t, 0 ; u(\cdot))$ follows immediately.

Remark 5.1. Clearly, if there exists a constant $\lambda>0$ such that

$$
\left\{\begin{array}{l}
G \geq 0, \quad G+\widehat{G} \geq 0, \quad R(s) \geq \lambda I, \quad R(s)+\widehat{R}(s) \geq \lambda I, \quad \text { a.e. } \quad s \in[t, T], \\
Q(s) \geq 0, \quad Q(s)+\widehat{Q}(s) \geq 0,
\end{array}\right.
$$

then the map $u(\cdot) \mapsto \mathcal{J}(t, 0 ; u(\cdot))$ is uniformly convex. This is a generalization of the standard LQ problem to the mean-field case. Similarly to the argument in ([16], Sect. 6), one can show that another case which guarantees the uniform convexity of the map $u(\cdot) \mapsto \mathcal{J}(t, 0 ; u(\cdot))$ is as follows:

$$
\left\{\begin{array}{l}
G \geq \lambda I, \quad G+\widehat{G} \geq \lambda I, \quad R(s), R(s)+\widehat{R}(s) \geq 0, \quad Q(s) \geq 0, \\
Q(s)+\widehat{Q}(s) \geq 0, \quad C(s)=\widehat{C}(s), \quad D(s)=\widehat{D}(s) \text { is surjective, }
\end{array} \quad \text { a.e. } \quad s \in[t, T],\right.
$$

However, until now, there is no good characterization for the uniform convexity of that map. This will be investigated in our future work.

\section{REFERENCES}

[1] B. Acciaio, J. Backhoff-Veraguas and R. Carmona, Extended mean field control problems: stochastic maximum principle and transport perspective. SIAM J. Control Optim. 57 (2019) 3666-3693.

[2] N. Agram and B. Øksendal, Stochastic control of memory mean-field processes. Appl. Math. Optim. 79 (2019) $181-204$.

[3] B.D.O. Anderson and J.B. Moore, Optimal Control: Linear Quadratic Methods. Prentice Hall, Englewood Cliffs, NJ (1989).

[4] A. Bensoussan, G. Da Prato, M.C. Delfour and S.K. Mitter, Representation and control of infinite-dimensional systems. Vol. II. Birkhäuser Boston, Inc., Boston, MA (1993).

[5] A. Bensoussan, J. Frehse and P. Yam, Mean field games and mean field type control theory. Springer, New York (2013).

[6] F.J. Beutler, The operator theory of the pseudo-inverse. II. Unbounded operators with arbitrary range. J. Math. Anal. Appl. 10 (1965) 471-493.

[7] P. Cardaliaguet, F. Delarue, J.-M. Lasry and P.-L. Lions, The master equation and the convergence problem in mean field games. Princeton University Press, Princeton, NJ (2019).

[8] R. Carmona and F. Delarue, Probabilistic theory of mean field games with applications. I. Mean field FBSDEs, control, and games. Springer, Cham (2018).

[9] R. Carmona and F. Delarue, Probabilistic theory of mean field games with applications. II. Mean field games with common noise and master equations. Springer, Cham (2018).

[10] G. Da Prato and J. Zabczyk, Stochastic Equations in Infinite Dimensions. Cambridge University Press, Cambridge (1992).

[11] D.A. Dawson, Stochastic evolution equations. Math. Biosci. 15 (1972) 287-316.

[12] R. Dumitrescu, B. Øksendal and A. Sulem, Stochastic control for mean-field stochastic partial differential equations with jumps. J. Optim. Theory Appl. 176 (2018) 559-584.

[13] G. Lance, E. Trélat and E. Zuazua, Shape turnpike for linear parabolic PDE models. Systems Control Lett. 142 (2020) 104733.

[14] X. Li, J. Sun and J. Yong, Mean-field stochastic linear quadratic optimal control problems: closed-loop solvability. To appear in: Probab. Uncertain. Quant. Risk doi: 10.3934/mcrf.2020026 (2020). 
[15] X. Li and J. Yong, Optimal control theory for infinite-dimensional systems. Systems \& Control: Foundations \& Applications. Birkhäuser Boston, Inc., Boston, MA (1995).

[16] Q. Lü, Well-posedness of stochastic Riccati equations and closed-loop solvability for stochastic linear quadratic optimal control problems. J. Differ. Equ. 267 (2019) 180-227.

[17] Q. Lü and X. Zhang, General Pontryagin-Type Stochastic Maximum Principle and Backward Stochastic Evolution Equations in Infinite Dimensions. Springer Briefs in Mathematics. Springer, Cham (2014).

[18] A. Porretta and E. Zuazua, Long time versus steady state optimal control. SIAM J. Control Optim. 51 (2013) $4242-4273$.

[19] J. Sun, Mean-field stochastic linear quadratic optimal control problems: open-loop solvabilities. ESAIM: COCV 23 (2017) 1099-1127.

[20] J. Sun, X. Li and J. Yong, Open-loop and closed-loop solvabilities for stochastic linear quadratic optimal control problems. SIAM J. Control Optim. 54 (2016) 2274-2308.

[21] T. Suzuki, Mean field theories and dual variation. Atlantis Press, Paris (2015).

[22] M. Tang, Q. Meng and M. Wang, Forward and backward mean-field stochastic partial differential equation and optimal control. Chin. Ann. Math. Ser. B 40 (2019) 515-540.

[23] J. B. Walsh, An introduction to stochastic partial differential equations. École d'été de probabilités de Saint-Flour, XIV-1984, 265-439, Lecture Notes in Math., 1180. Springer, Berlin (1986).

[24] T. Wang, Necessary conditions in stochastic linear quadratic problems and their applications. J. Math. Anal. Appl. 469 (2019) 280-297.

[25] T. Wang, On closed-loop equilibrium strategies for mean-field stochastic linear quadratic problems. ESAIM: COCV 26 (2020) 41.

[26] Q .Wei, J. Yong and Z. Yu, Linear quadratic stochastic optimal control problems with operator coefficients: open-loop solutions. ESAIM: COCV 25 (2019) 17.

[27] J. Yong, Linear-quadratic optimal control problems for mean-field stochastic differential equations. SIAM J. Control Optim. 51 (2013) 2809-2838.

[28] J. Yong, Linear-quadratic optimal control problems for mean-field stochastic differential equations-time-consistent solutions. Trans. Amer. Math. Soc. 369 (2017) 5467-5523.

[29] J. Yong and X.Y. Zhou, Stochastic Controls: Hamiltonian Systems and HJB Equations. Springer-Verlag, New York (1999). 